\section{DR.264}

UCRL-53199

$y^{\prime}$ Shorting Time of Magnetically Insulated Reflex-Ion Diodes

from the Neutral-Atom Charge-

Exchange Mechanism

George Strobel

MASTER

October 1981

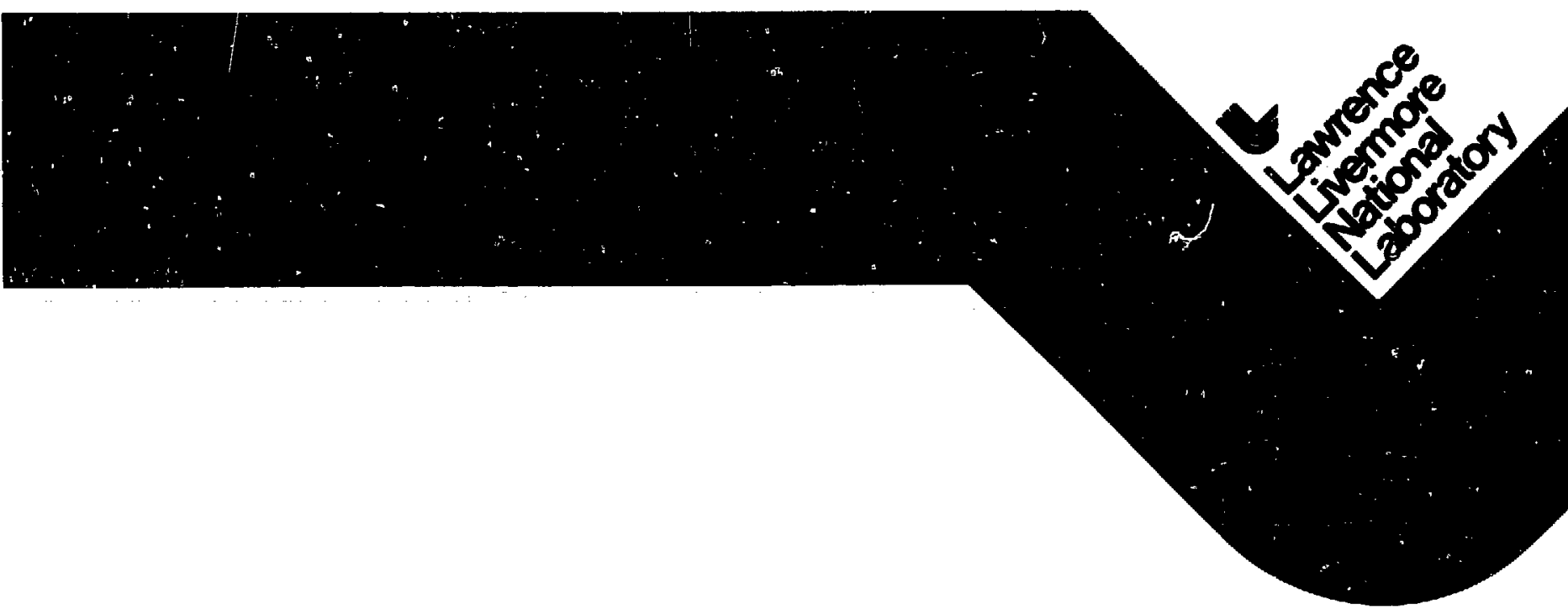


UCRL-53199

Distribution Category UC-20

UCRL- -53199

DE82 007733

\title{
Shorting Time of Magnetically Insulated Reflex-Ion Diodes from the Neutral-Atom Charge- Exchange Mechanism
}

\author{
George Strobel*
}

Manuscript date: October 1981

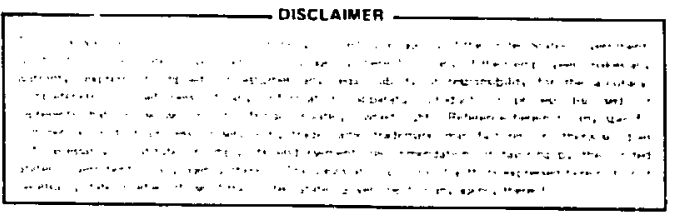

"Department of Physics and Astronomy, University of Georgia, Athens, Georgia.

\section{LAWRENCE LIVERMORE LABORATORY University of California $\bullet$ Livermore, California 94550}




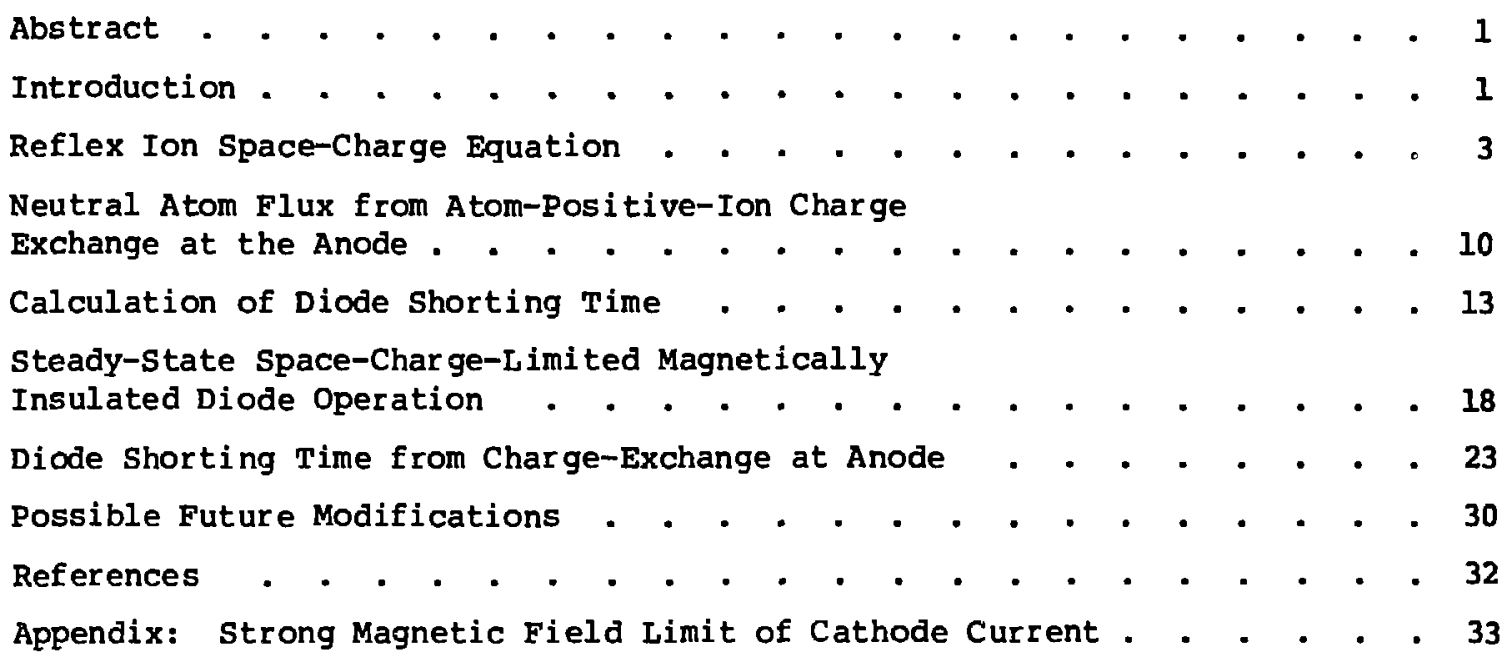


SHORTING TIME OF MAGNETICALLY INSULATED REFLEX-ION DIODES

FROM THE NEUTRAL-ATOM CHARGE-EXCHANGE MECHANISM

ABSTRACT

In a magnetically insulated diode, collision-free electrons return to the cathode and no electron current is present at the anode. Electron transport to the anode is studied in this paper. Steady-state space-charge-limited flow is assumed initially. Breakdown of ion flow occurs when static neutral atoms at the anode undergo charge exchange, which results in neutral atoms drifting across the diode. These are subsequently ionized by reflexing ions producing electrons trapped in Larmor orbits throughout the diode. These electrons drift to the anode via ionization and inelastic collisions with other neutral atoms.

Model calculations compare the effects of foil and mesh cathodes. steadystate space-charge-limited ion current densities are calculated. The neutral atom density at the cathode is determined as a function of time. The shorting time of the diode is scaled versus the electrode separation $d$, the diode potential $v_{0}$, the magnetic field, and the initial concentration of static neutral atoms. The neutral atom flux at the cathode is found to equal the ion flux at the anode in $10(d / c m)$ nanoseconds, where $d$ is the diode separation. The neutral atom density at the cathode reaches its equilibriun value in about 100 ( $d / c m$ ) nanoseconds. The diode shorting time varies as $d^{2} N_{0}$, and is exponentially dependent on the initial neutral atom concentration.

\section{INTRODUCTION}

We consider the one-dimenstonal magnetically insulated reflex ion diode. ${ }^{1-4}$ Electron motion is treated relativistically. We use cylindrical coordinates $r, \theta, z$. Azimuthal symmetry is assumed, with the electric field parallel to the $z$ axis, an anode at the $z=0$ plane, a cathode at the $z=d$ plane. A separate current along the $z$ axis creates an azimuthal magnetic $f$ ield that falls off with $r$, the radial distance from the $z$ axis. This magnetic field is so strong that electrons emitted at the cathode cannot reach the anode, but return instead to the cathode. The cathode is a thin foil or mesh. The positive ions traverse the cathode, returning through it several times 
before losing all their energy. Curvature of the ion motion in the magnetic field is neglected. We take the potential to be a function of $z$ only. We reglect any radial dependence and solve a one-dimensional problem.

In crossed uniform electric and magnetic flelds, the collision-free orbit of an electron has a cycloidal shape. The electron starts from rest at the cathode. Provided the magnetic field exceeds a critical strength $B$, the electron advances a maximum distance $b$ towards the anode, and turns back toward the cathode.

The key factor in shorting a magnetically insulated diode is the deviation of electrons from collision-free paths, such that they fail to return to the cathode. One mechanism for electron drift across a diode is provided by largeangle coulombic collisions, which can produce a noticeable shift towards the anode of the center of an electron's orbit. That mechanism is not considered here. A second mechanism proceeds as follows: Ionization of a neutral atom by the orbiting electron produces a second electron, whose subsequent orbit lies a significant fraction of $b$ closer to the anode than did the orbit of the initiating electron. In this work, we consider how the ionization and inelastic scattering from neutral deuterium atoms may promote electron transit across a magnetically insulated disde of width $d$.

Qualitatively, if the electron transit tine across the diode gap is comparable to the positive ion transit time, then desired operation of the diode has broken down and we say the diode is shorted. This electron transit time depends sensitively on the neutral atom concentration $\mathrm{N}_{\mathrm{g}^{\prime}}$ via the mean free path for electrons to ionize a neutral atom. The neutral atom concentration at the cathode varles with time. We will apply a model developed by $D$. $S$. Prono, H. Ishizuka, E. P. Lee, B. พ. Stallard, and พ. C. Tuzner 5 to determine $\mathrm{N}_{\mathrm{g}}$ as a function of time. In this model lons at the anode are accelerated towards the cathode. Charge exchange occurs between the lons and static neutral atoms in the anode vicinity, resulting in moving neutrals that subsequently arrive at the cathode. The neutral crossing time depends on the initial distribution of the static neutrals and also on the ion currents in the diode. The ion currents are calculated assuming space-charge-limited flow at both the anode and the cathode. The radial variation of the magnetic field and the electric potential is neglected, resulting in a one-dimensioval problem that is solved numerically. The static neutral atoms are assumed to be uniformly concentrated at the anode for a thickness of $0.1 \mathrm{~cm}$. The shorting time of the diode is calculated as a function of various initial 
static neutral concentrations, and of the diode potential and magnetic field.

Diodes short with the following assumed scenario of events: The diode is instantaneously put into steady-state space-charge-limited ion flow, with electron flow restricted to the cathode region by a given applied magnetic field. Charge exchange between ions and static neutrals occurs, producing neutral atoms which drift across to the cathode. The neutral atoms are subsequently ionized by reflexing ions, resulting in electrons trapped in Larmor orbits throughout the diode. These electrons ionize and inelastically scatter from more neutral atoms resulting in momentarily static electrons. These electrons are accelerated towards the anode in a Larmor orbit. We define the diode as shorted when the electron current density at the anode equals the ion current density.

\section{REFLEX ION SPACE-CHARGE EQUATION}

The starting equations are Poisson's equation for the potential, conservation of energy for the ions and electrons, conservation of momentum for electrons in the radial direction, and a reflex ion energy distribution at the cathode. We will determine the potential assuming the electron motion is collision free.

In terms of $n$, the electron density: $N_{0}$, the density of positive ions that have never traversed the cathode; and $N$, the reflex ion density, Poisson's equation is

$$
\frac{d^{2} \phi}{d z^{2}}=4 \pi e\left(n-N-N_{0}\right) .
$$

The electric potential is $\phi$, and is assumed to be a function of $z$ only, hence the absence of radial and azimuthal derivatives in Eq. (1). The electric field is given by $E=-d \phi / d z$. The boundary conditions on the potential for space-charge-limited flow are that

at the anode $\phi=v_{0}$, and $\frac{d \phi}{d z}=0$; at the cathode $\phi=0$, and $\frac{d \phi}{d z}=0$.

We will use the relation that the current density $j$ is equal to the velocity times the charge density. In steady state, by charge conservation, $j$ must be 
independent of $z$. This together with conservation of energy and momentum will evolve expressions for the right hand side of $\mathrm{Eq}$. (1) that are known functions of $z$, of the magnetic field, and of $J$ and $j$ (which are, respectively, the anode ion current density and the cathode electron current density). Though one-dimensional motion is assumed throughout, these quantities will depend on $r$, as the magnetic field $B$ depends on $r$. Equation $(1)$ can be integrated once analytically to obtain an exact equation for the electric field in the vicinity of the anode. We will. determine the ion and electron current density $J$ and $j$ from the resulting first-order differential equations.

In the vicinity of the cathode, an approximation is required to obtain the electric field analytically, as electron path curvature is included. Figure 1 shows the path of a typical ion and a typical electron. The ions are all assumed to be singly ionized; $e$ is the proton charge, $M$ the ion mass, and $m$ the electron mass. The electrons are assumed to be produced at rest at the cathode, and the ions are produced at rest at the anode. Conservation of energy results in

$$
\frac{1}{2} M v^{2}=e v_{0}-e \phi
$$

for the ion kinetic energy, and

$$
m c^{2}(\gamma-1)=e \phi
$$

for the electron kinetic energy, where

$$
\gamma^{2}=1 /\left[1-(v / c)^{2}\right]
$$

and $c$ is the speed of light.

We define the azimuthal component of the magnetic field as

$$
B=\frac{B_{0}}{C r},
$$

where $r$ is the radial distance and $B_{0}$ is a constant. Then conservation of canonical mamentum yields

$$
\gamma m v_{r}=\frac{B_{0}}{c r}(z-d) \equiv A \text {, }
$$

where $A$ is the magnitude of the vector potential. 


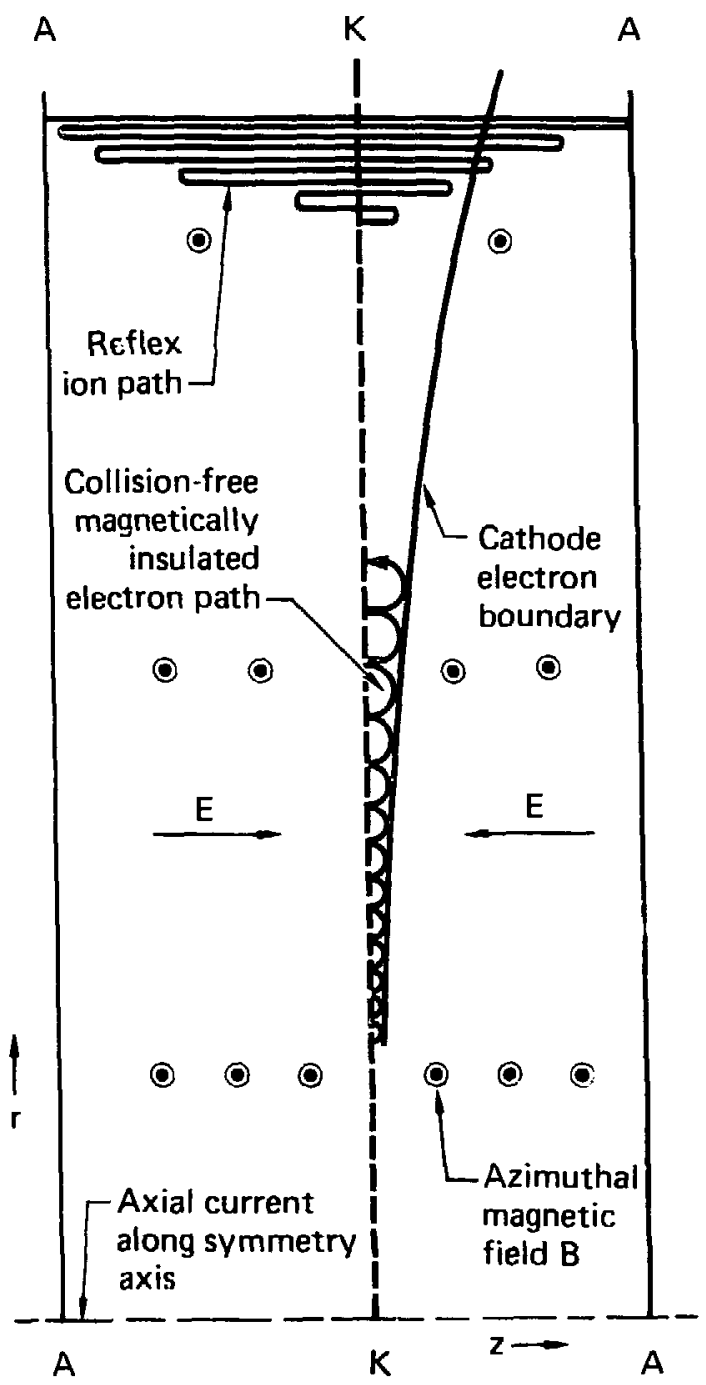

Fig. 1. Geometry of the axially symmetric magnetically insulated ion diode. The cathode is a mesh or foil labeled $K$, and the anode is labeled $A$. An axial current gees down the center line, producing an azimuthal magnetic field that bends the path of the electrons into a cycloid. The positive ions reflex several times through the cathode before ending at the cathode.

Using Eq. (5) in Eq. (7), we have

$$
v_{r}^{2}=\frac{A^{2}}{m^{2}+A^{2} / c^{2}}\left[1-\left(v_{z} / c\right)^{2}\right] \text {. }
$$

Substituting Eq. (5) and Eq. (8) into Eq. (4) results in

$$
\left(v_{z} / c\right)^{2}=1-\frac{A^{2} c^{2}+m^{2} c^{4}}{\left(m c^{2}+e \phi\right)^{2}}
$$




$$
\text { en }=\frac{2 j}{v_{z}}=\frac{2 j}{c\left[1-\frac{A^{2} c^{2}+m^{2} c^{4}}{\left(m c^{2}+e \phi\right)^{2}}\right]^{1 / 2}}=\frac{2 j\left(1+e / m c^{2}\right)}{c\left[\frac{2 e \phi}{m c^{2}}+\frac{e^{2} \phi^{2}}{m^{2} c^{4}}-\frac{B_{0}^{2}(z-d)^{2}}{r^{2} m^{2} c^{4}}\right]^{1 / 2}} .
$$

Thus the electron density has been expressed in terms of the current density $j$ leaving the cathode, the potential $\phi$ within the gap, and the magnetic field B within the gap. The factor of 2 comes from all the electrons returning to the cathode. Equation (10) for the electron density contributes to Eq. (1) only where the electrons exist. If the radical in Eq. (10) is negative, then the electrons cannot exist there, and the entire term $\mathbf{n}$ is omitted from Eq. (1). The initial ions $N_{0}$ can exist everywhere in the gap $(0<z<d)$ as their curvature is ignored. The reflex ion distribution $\mathrm{N}$ is limited depending on the energy distribution assumed at the cathode.

We now consider the reflex ion energy distribution. We introduce a reflex ion energy distribution $f$. This is the energy distribution of ions that have passed through the cathode boundary. This distribution is normalized in terms of $\mathrm{J}$, the anode ion current density. The maximum energy of the reflex ion distribution is $v_{0}$ from energy considerations, but since ions lose energy by passing through the cathode and its electron sheath, the ion energy distribution must extend down to zero eriergy. Let eT = energy, so $T$ represents a (variable) potential. At the anode, the average number of times an ion is incident on the anode is

$$
n=1+\int_{0}^{V_{0}} f(T) d T
$$

The reflex ion density can be expressed in terms of $f$ as

$$
e N=2 J \int_{\phi}^{V_{0}} \frac{f(T) d T}{\left[\frac{2 e}{M}(T-\phi)\right]^{1 / 2}}
$$

The factor $J$ results from the reflex ion distribution being normalized in terms of $J_{;}$that is, $f$ is the distribution at the cathode per unit ion-current 
emitted at the anode. The factor of 2 in Eq. (12) comes because every reflex ion is reflected at some point in the diode, where $T=\phi(z)$. The reflex density $\mathrm{N}$ varies with position because the potential $\phi$ varies with position $z$. At a given location $z$, where the potential is $\phi(z)$, only those ions whose energy at the cathode $[e T \geq e \phi(z)]$ was at least e $\phi(z)$ will contribute to the reflex ion density $\left(\right.$ at $z$ ). For $\mathrm{N}_{0}$, the density of ions on their initial pass from the anode to the cathode, we have

$$
\mathrm{eN}_{0}=\frac{\mathrm{J}}{\mathrm{v}_{\mathrm{z}}}=\frac{\mathrm{J}}{\left[\frac{2 \mathrm{e}}{\mathrm{M}}\left(\mathrm{v}_{0}-\phi\right)\right]^{1 / 2}},
$$

where $J$ is the ion current density emitted at the anode. Usually, with the assumption of one-dimensional motion, $J$ will be independent of the other two coordinates. However, with the reflex magnetically insulated diode, we will find $J$ depends on the radial coordinate $r$ even though the potential at $z=0$ and at $z=d$ is independent of $(r, \theta)$. This radial dependence of $J$ comes from the magnetic field $B$ depending on $r$. We seek relations of $J$ and $j$, the ion and electron current densities, for various reflex energy distributions E. We are able to solve for $J$ and for $j$ as we have four boundary ccnditions, see Eq. (2), to apply. Two boundary conditions are required to solve Eq. (1), which is a second-order differential equation; the other two boundary conditions determine $\mathrm{J}$ and $\mathrm{j}$. It is convenient to normalize the current densities in terms of the Child-Langmuir current density:

$$
\begin{aligned}
& j_{0}=\varepsilon_{0} \frac{4}{9} \sqrt{\frac{2 \mathrm{e}}{\mathrm{m}}}\left(\mathrm{v}_{0}\right)^{3 / 2} / \mathrm{d}^{2}, \\
& J_{0}=\varepsilon_{0} \frac{4}{9} \sqrt{\frac{2 \mathrm{e}}{M}}\left(\mathrm{v}_{0}\right)^{3 / 2} / \mathrm{d}^{2} .
\end{aligned}
$$

The electron's mass is represented by $m$; the ion mass, by $M$.

It is convenient to introduce dimensionless coordinates:

$$
\begin{array}{lll}
\mathrm{x}=\mathrm{z} / \mathrm{d} & \text { so } & 0 \leq \mathrm{x} \leq 1 \\
\mathrm{u}=\phi / \mathrm{N}_{0} & \text { so } & 0 \leq \mathrm{u} \leq 1 \\
\xi=\mathrm{T} / \mathrm{N}_{0} & \text { so } & 0 \leq \xi \leq 1 .
\end{array}
$$


Then substituting Eqs. (10-13) into Eq. (1), we have

$$
\begin{aligned}
\frac{\mathrm{d}^{2} \mathrm{u}}{\mathrm{dx^{2 }}} & =-\frac{J}{J_{0}} \frac{1}{\sqrt{l-u}}-\frac{2 J}{J_{0}} \int_{u}^{1} \frac{v_{0} f(\xi) d \xi}{\sqrt{\xi-u}} \\
& +\frac{2 j\left(1+\frac{e v_{0}}{m c^{2}} u\right)}{j_{0}\left[u+\frac{e v_{0}}{2 m c^{2}} u^{2}-\frac{B_{0}^{2}(x-1)^{2} d^{2}}{r^{2} m c^{2} 2 e v_{0}}\right]} .
\end{aligned}
$$

Now the electrons contribute to Eq. (16) only where the radical in the last term is non-negative. As $x$ approaches zero, $u$ approaches 1 , and this radical goes negative at the electron cutoff boundary, called $x=b$. In terms of the normalized potential at the cutoff of electron density, $U=u(b)$, we have, from setting the radical in Eq. (16) to zero,

$$
b=1-\frac{r}{B_{0} d} \sqrt{2 m c^{2} e v_{0}}\left[u+\frac{e v_{0} v^{2}}{2 m c^{2}}\right]^{1 / 2},
$$

as the boundary for electron collision-free motion near the cathode. For the magnetically insulated diode, $0<b \leq 1$. The critical magnetic field is found from Eq. (17) by setting $b=0, r$ to $r_{m x}$, and $u=1$.

For certain simple distributions $f$, we can construct analytic first integrals of Eq. (16). 6 Now define

$$
s(u) \equiv \int_{u}^{l} d v \int_{v}^{l} \frac{f(\xi) d \xi}{\sqrt{\xi-v}}
$$

where $S(u)$ is the number of reflex ions between the anode and the point where the potential is $u$. Then integrating $\mathrm{Eq} .(16)$ by the factor

$$
\int 2 \frac{d u}{d x} d x
$$

results in

$$
\left(\frac{d u}{d x}\right)^{2}=\frac{4 J}{J_{0}}(\sqrt{1-u}+s(u)) .
$$


Thus the electric field in the anode region is given by the square root of Eq. (19), and $u$ as a function of $x$ is found from

$$
x=\int_{u}^{1} \frac{d u}{\left[\frac{4 J}{J_{0}}(s(u)+\sqrt{1-u})\right]^{1 / 2}} .
$$

For the region $b \leq x \leq 1$ near the ca hode, we have

$$
\begin{aligned}
& \left(\frac{d u}{d x}\right)^{2}=-\frac{4 J}{J_{0}}[1-\sqrt{1-u}+s(0)-s(1)] \\
& +\frac{4 j}{j_{0}} \int_{0}^{u(x)} \frac{d u\left(1+e v_{0} u / m c^{2}\right)}{\left[u+\frac{e v_{0}}{2 m c^{2}} u^{2}-\frac{(x-1)^{2}}{(1-b)^{2}}\left(u+\frac{e v_{0}}{2 m c^{2}} u^{2}\right)\right]^{1 / 2}} ;
\end{aligned}
$$

where we have used Eq. (17) to obtain Eq. (21). We cannot evaluate the integral in Eq. (21) as it stands since we must know $x$ as a function of $u$, or conversely, $u$ as a function of $x$. That is precisely the problem we want to solve. This problem arises only in the cathode region of the magnetically insulated reflex ion diode.

We approximate the last term of the radical in Eq. (2I) as

$$
\frac{(1-x)^{2}}{(1-b)^{2}}=A\left(\frac{u}{U}\right)^{3 / 2}+(1-A)\left(\frac{u}{U}\right)^{2} \text {, }
$$

where $A$ is a parameter determined from $S(u)$. Substituting Eq. (22) into Eq. (2l) and integrating for small $u$ results in a quadratic equation that can be solved for $A$; see the appendix for some details.

Inserting Eq. (22) into Eq. (21) we have for arbitrary U:

$$
\left(\frac{d u}{d x}\right)^{2}=-\frac{4 J}{J_{0}}[1-\sqrt{1-u}+s(0)-s(u)]+\frac{4 j}{j_{0}} I(u, A, U),
$$

where

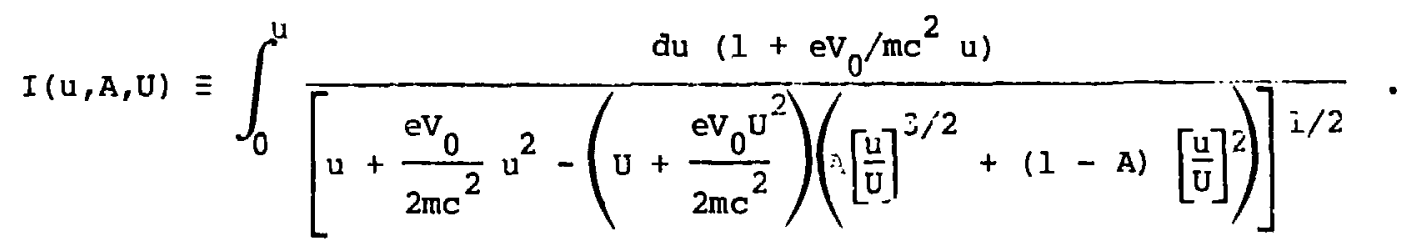

This integral can be analytically evaluated. Note $U$ is a constant. 
Equations (1.9) and (23) are solved numerically and the cathode and anode curren't densities $j$ and $J$ are found by requiring the electric fields to be continuous at the electron turning point b. The turning point $b$ is found by reguiring the potential to be continuous at the turning point.

\section{NEUTRAL ATOM FLUX FROM ATOM-POSITIVE-ION CHARGE} EXCHANGE AT THE ANODE

The neut, al atom flux is determined by a charge exchange mociel developed by Prono et al. ${ }^{5}$ In this model a static neutral atom concentration $n_{A}$ is assumed to exist near the anode. Initially no moving neutrals exist anywhere, and the neutral atoms are $i$ the region $0<x<x_{\max } x_{\max }$ is a parameter set to $0.1 \mathrm{~cm}$ here. At $t=0$, the steady-state space-charge-limited positive ion current begins passing through these static neutral atoms. Some of the positive ions undergo charge exchange, resulting in drifting neutral atoms and in static ions that are accelerated to repeat the process. This model treats the electric field $\mathrm{E}$ as constant over the region of the initial neutral atom concentration. The energy dependence of the ion-neutral-atom charge-exchange cross section shown ${ }^{7}$ in Fig. 2 is modeled as

$$
\sigma=\sigma_{0} /\left(1+w^{2} / w_{0}^{2}\right)
$$

where $w$ is the ion energy, $w_{0}=10 \mathrm{keV}$, and $\sigma_{0}=2 \times 10^{-15} \mathrm{~cm}^{2}$.

The result of the model is a distribution function $g$ of drifting neutral atoms given by ${ }^{5}$

$$
g=\frac{\alpha M F}{C w_{0}} e^{-B \alpha W} H(x-\alpha W) \quad\left[1-e^{-C B(X-\alpha W)} / B\right] \text {. }
$$

where

$$
\begin{aligned}
& \alpha=n_{A} \sigma_{0} \omega_{0} / e E, \\
& B=\frac{1}{1-c}, \\
& C=e^{-\alpha \pi / 2},
\end{aligned}
$$



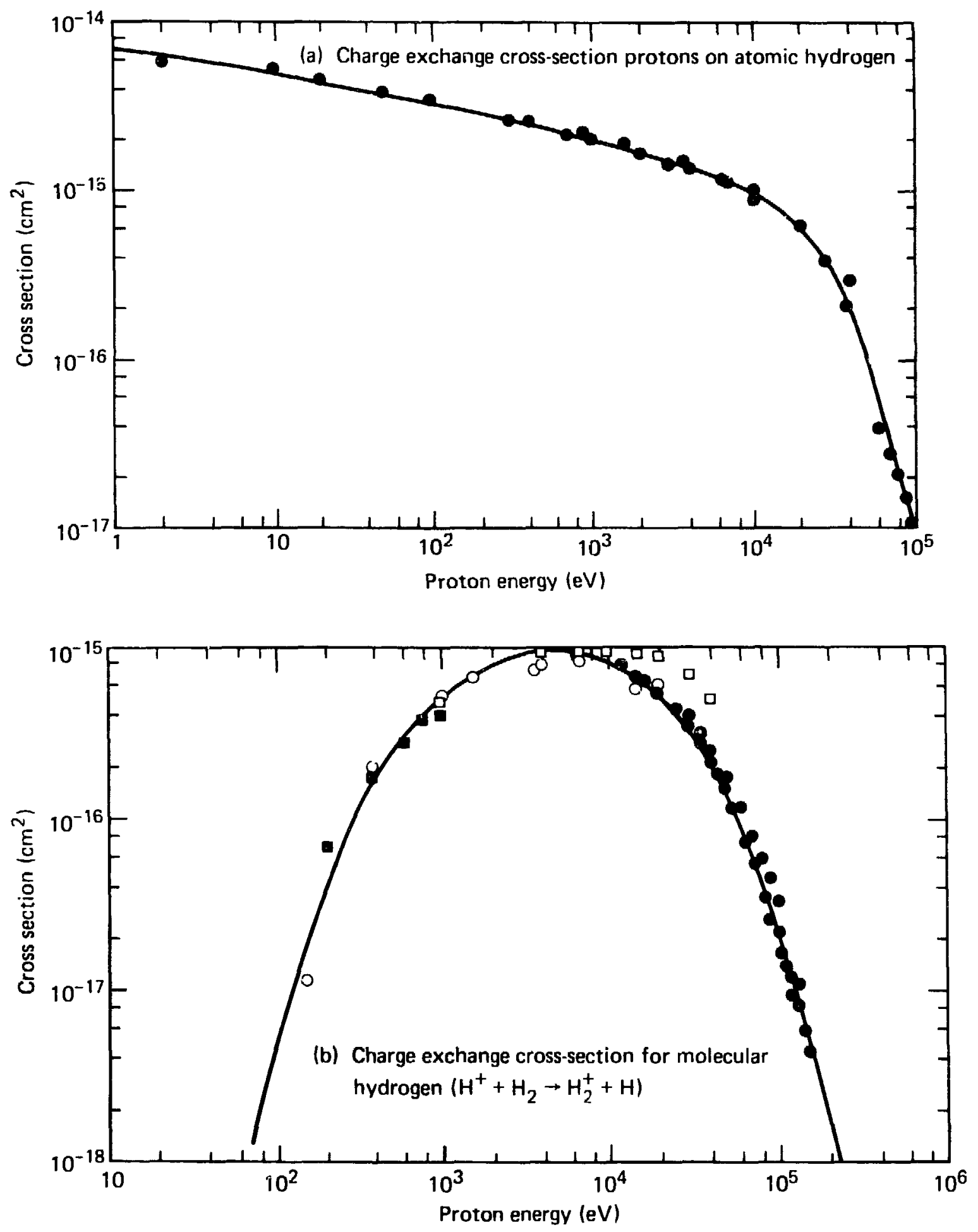

Fig. 2. Proton-induced charge-exchange cross sections on (a) atomic, and (b) molecular, hydrogen. The data shown here were compiled from various sources. For more detail, see Ref. 7 . 
$F$ is the steady-state ion flux leaving the anode, $H$ is the unit step function, equal to zero (one) for negative (positive) argument, and $w$ and $x$ are normalized energies and locations given by

$$
w=w / w_{0}
$$

and

$$
x=n_{A} \sigma_{0} x
$$

In Eqs. (26-37), B is a model parameter, given in Eq. (28), and not the magnetic field.

We assume these neutral atoms drift without interaction across the diode after leaving the static central atom concentration. This neglects any further charge exchange between drifting neutrals and reflexins ions. Charge exchange between the drifting neutral and a reflex ion would slow the drift of neutrals across the diode.

The number of neutrals that have crossed the diode will now be calculated from the distribution function $\mathrm{g}$. This number can be obtained as a function of time. When the neutral concentration at the cathode is large enough for collisionless electron flow to break down, we say the diode has shorted out.

The number of neutral atoms per $\mathrm{cm}^{2}$ that originated at the anode and are present a distance $d$ beyond the boundary of the original neutral concentration is given by

$$
\mathscr{N}=\int_{d / t}^{\infty} v g[t-d / v] d v=\int_{d / t}^{\infty} g[v t-d] d v .
$$

Using Eq. (26) in Eq. (32), the integrals can be evaluated in terms of tabulated functions. The result is:

$$
\begin{gathered}
\mathscr{N}=\frac{F t}{B C}\left\{e^{-B \alpha L}-e^{-B X}-e^{-C B X}\left[e^{-\alpha L}-e^{-X}\right]\right\} \\
-\frac{F d}{B C} \sqrt{\frac{\alpha M \pi}{2 w_{0}}}\left\{\sqrt{B}[\operatorname{erf}(\sqrt{B X})-\operatorname{erf} \sqrt{B \alpha L}]-e^{-C B X}[\operatorname{erf}(\sqrt{X})-\operatorname{erf}(\sqrt{\alpha L})]\right\},
\end{gathered}
$$

where $L=M a^{2} / 2 w_{0} t^{2}$, and $M$ is the ion mass. 
In Eq. (33), $t$ is restricted to $t>d \frac{\alpha M}{2 W_{0} x}$

This restriction is the time for the most energetic neutral atom to cross the diode. If we assume a uniform electric field in the diode, with a potential of $2 \mathrm{MeV}$, that the ions are deuterons, and that the neutral atoms extend 98 of the way from the anode to the cathode, then the minimum time is

$$
t_{0}=\frac{d}{c} \sqrt{\frac{M c^{2} d}{2 e V_{0} X}}=\frac{d}{c} \sqrt{\frac{3672}{2.2(0.09)}} \approx \frac{d}{c} 10^{2} \text {. }
$$

This minimum time is about $3 \mathrm{~ns}\left(3 \times 10^{-9} \mathrm{sec}\right)$ for $\mathrm{d}=1 \mathrm{~cm}$. The restriction of $\mathrm{Eq}$. (34) also implies $\alpha \mathrm{L}<\mathrm{X}$.

An approximation of Eq. (33), valid for large times for the flux is

$$
\mathrm{G} \approx \frac{\mathrm{F}}{\mathrm{BC}}=\mathrm{F}\left[\exp \left(\frac{{ }_{\mathrm{a}} \sigma_{0}{ }^{w_{0}} \pi}{2 \mathrm{eE}}\right)-1\right]
$$

The flux $G$ of neutral atoms at the cathode is the first line of Eq. (33) divided by t. To use Eq. (35) or Eq. (33) we write the space-charge-limited ion flux as

$$
F=\frac{J}{J_{0}}\left(\frac{J_{0}}{e}\right)=\left(\frac{\mathrm{J}}{J_{0}}\right) 1.7 \times 10^{20}\left(\frac{\mathrm{eV}_{0}}{\mathrm{MeV}}\right)^{3 / 2} \frac{1}{\left(\frac{\mathrm{d}}{\mathrm{cm}}\right)^{2}} \frac{\text { ions }}{\mathrm{cm}^{2}-\mathrm{sec}} .
$$

Thus, typical anode fluxes are of the order of $10^{20} \frac{\text { ions }}{\mathrm{cm}^{2}-\mathrm{sec}}$.

\section{CALCULATION OF DIODE SHORTING TIME}

We define the electron avalanche current density as

$$
j_{\mathbf{a}}=\frac{\text { neb }}{\mathrm{T}} \mathrm{P}_{\mathrm{t}}
$$

where $\mathrm{n}$ is the electron density as a function of time, $b$ is the scale of the Larmor orbits in the magnetic field, $T$ is the transit time for an electron to go one orbit, and $\mathrm{P}_{t}$ is the probability that an electron undergoes a large-angle interaction during a single Larmor orbit. If this has occurred, the electron can move a distance of order $b$ farther from the cathode. 
The shorting time is found by equating the electron avalanche current density to the positive ion current density. When these are equal we say the diode operation has broken dcwn.

The electron density $\mathrm{n}$ is calculated from solving the differential equation

$$
\frac{\mathrm{dn}}{\mathrm{dt}}=\mathrm{GP}^{\circ},
$$

where $G$ is the time-dependent neutral atom flux in the diode and $P^{0}$ is the probability of ionizing a neutral atom per centimeter path length of the neutral.

Including the ionization of neutrals by ions, by reflexing ions, and by electrons, we have

$$
P^{0}=\left\{1-\exp \left[-\int_{x}^{x+\Delta x}\left(n \sigma_{e}+N^{+} \sigma_{i}\right) d x\right]\right\}^{\prime} \Delta x
$$

When the ionization probability is small, we have for the rate of increase of the electron concentration

$$
\frac{d n}{d t}=G\left(n \sigma_{e}+N^{+} \sigma_{i}\right)
$$

where $\mathrm{N}^{+}$is the concentration of positive ions, $\sigma_{i}$ is the cross section for ionizing neutrals by positive ions, 7 and $\sigma_{e}$ is the cross section for ionizing neutrals by electrons. 7 See Figs. 3 and 4 for the energy-dependent cross sections. These cross sections have been energy averaged for use in Eq. (40) .

The neutral atom flux can be found from Eq. (33) as

$$
G=\frac{F}{B C}\left\{e^{-B \alpha L}-e^{-B X}-e^{-B C X}\left(e^{-\alpha L}-e^{-X}\right)\right\}
$$

where $L=M d^{2} / 2 w_{0} t^{2}$. For large times, and large $X$, this flux approaches

$$
G=F\left(e^{\alpha \pi / 2}-1\right)\left\{1-e^{-x\left[e^{\alpha \pi / 2}-1\right]}\right\}=F\left(e^{\alpha \pi / 2}-1\right)=F / B C .
$$



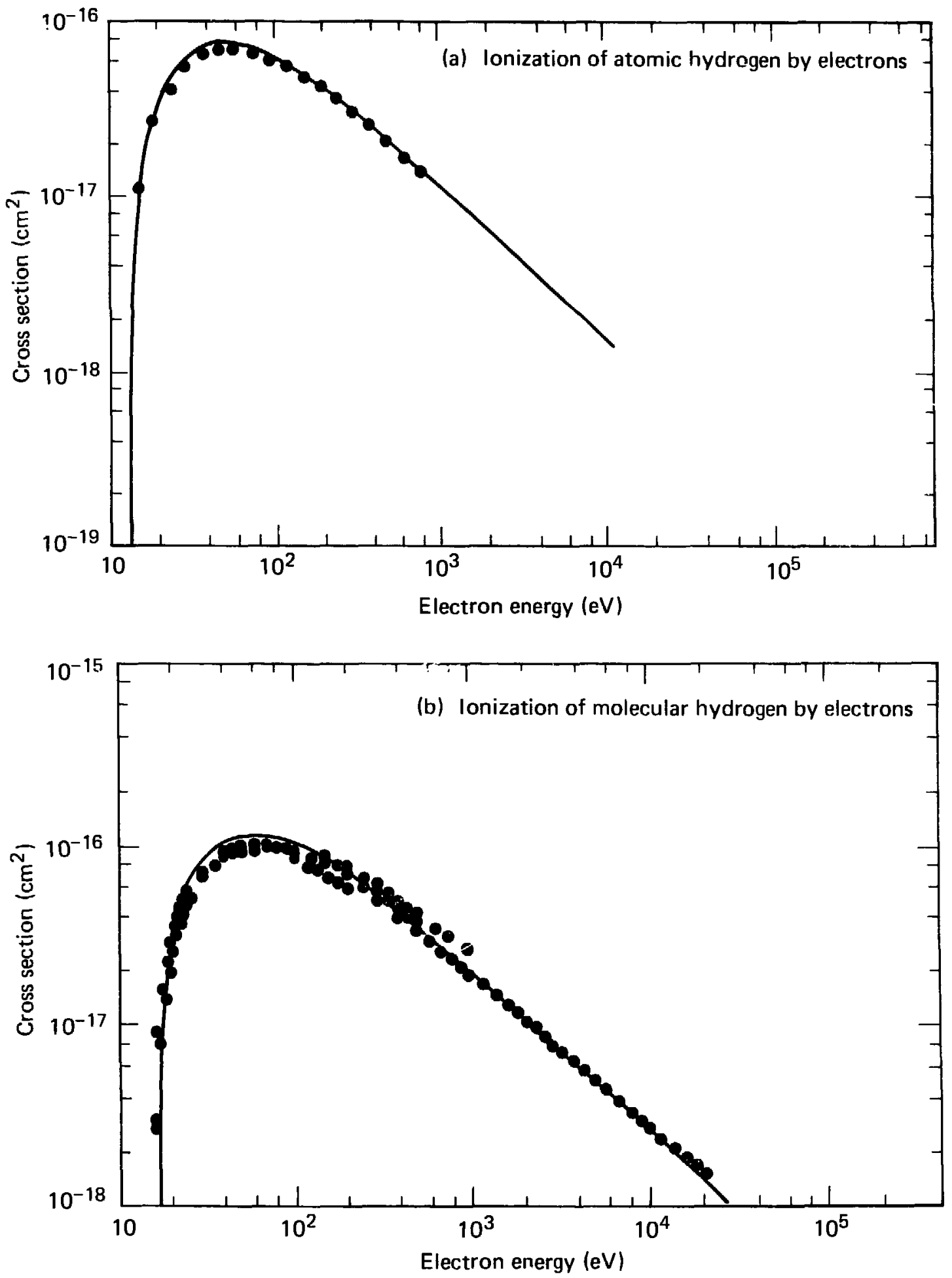

Fig. 3. Electron ionization cross sections of (a) atomic, and (b) molecular, hydrogen. The data shown here were compiled from yarious sources. For more detail, see Ref. 7. 

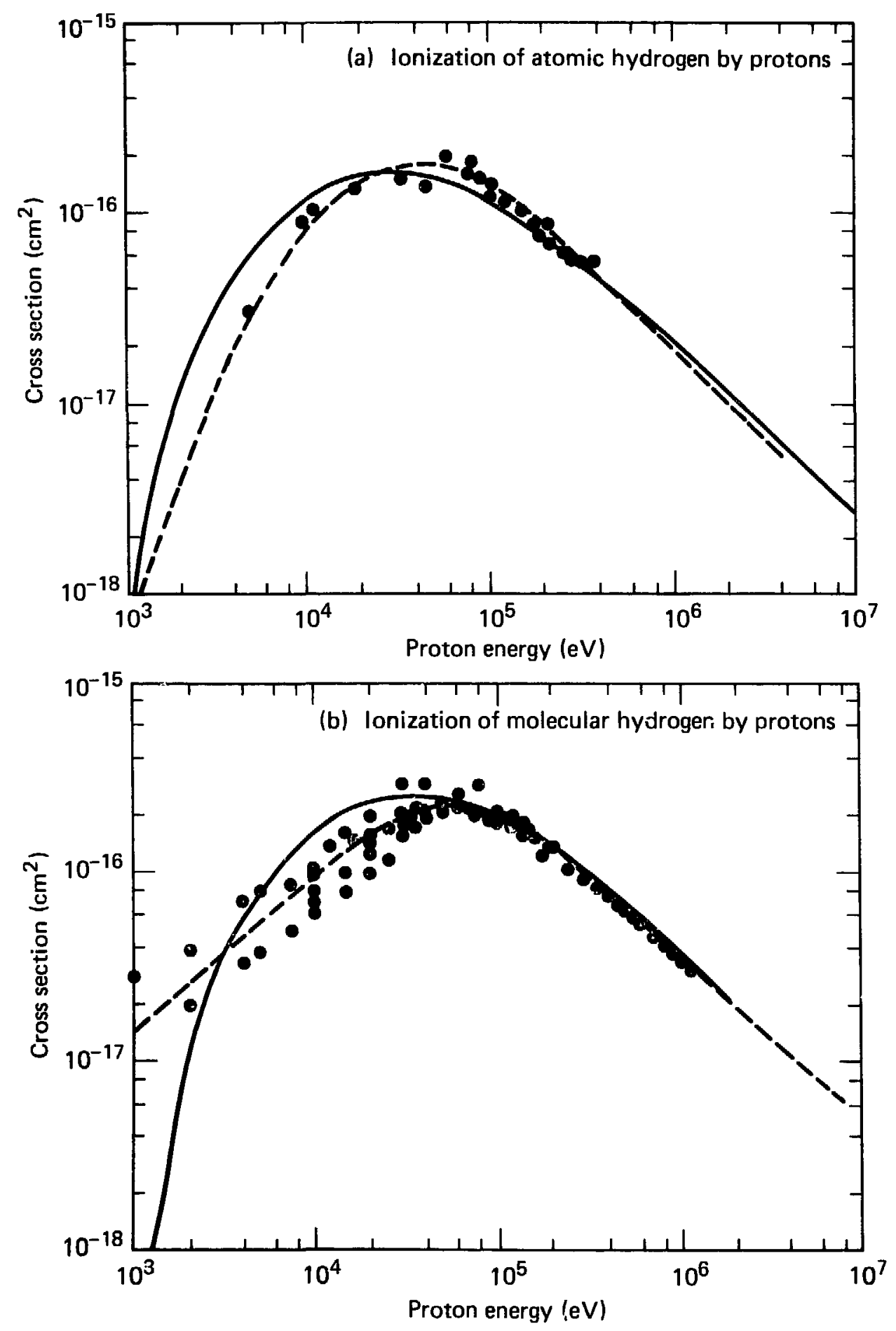

Fig. 4. Proton-induced ionization cross sections of (a) atomic, and (b) molecular, hydrogen. The data shown here were compiled from various sources. For more detail, see Ref. 7. The solid curves are predicted by theory; the broken curves represent a practical fit to the data. 
Using this asymptotic limit for the neutral flux, an analytic solution may be found for $n(t)$. Arguments based upon this approximation help to explain the qualitative behavior of the shorting time.

with $n(t=0)=0$, the solution to

$$
\frac{d n}{d t}=\left(n \sigma_{e}+N^{+} \sigma_{i}\right) F\left[e^{\alpha \pi / 2}-1\right]
$$

is

$$
n(t)=\frac{N^{+} \sigma_{i}}{\sigma_{e}}\left[e^{\sigma^{F t / B C}}-1-\sigma_{e} F t / B C\right] \text {. }
$$

Equating the electron avalanche current density, Eq. (37), to the positive ion current density eF, and using Eq. (43), we have for the shorting time $t_{s}$ '

$$
\frac{N^{+} \sigma_{i}}{\sigma_{e}}\left[e^{\sigma_{e} e^{F} / B C}-1-\sigma_{e} F t_{s} / B C\right] \frac{e b}{T}\left(3 N^{\circ} \sigma_{e} b\right)=e F \text {. }
$$

The large-angle electron cross section in Eq. (44) has been set to three times the electron-neutral ionization cross section because ionization is about one third of the total cross section. $N^{\circ}$ is the density of neutral atoms for asymptotically large times.

Defining:

$$
\tau \equiv \sigma_{e} F t_{s} / B C \equiv \sigma_{e} F t_{s}\left(e^{\sigma \pi / 2}-1\right)
$$

Eq. (44) can be written

$$
\left(e^{\tau}-1-\tau\right)=\frac{F \sqrt{\frac{2 m b d}{e v_{0}}}}{N^{+} \sigma_{i} 3 N_{0} b^{2}}
$$

and solved for $\tau$ numerically.

The shorting time found from Eqs. (45) and (46) is a function of magnetic field strength and the assumed initial neutral atom concentration, via the parameter $\alpha_{i}$ see Eq. (27). Equation (43) shows that the electron density in 
the diode rises initially as $t^{2}$, but eventually rises exponentially with a time constant $\mathrm{BC} / \mathrm{F \sigma}_{\mathrm{e}}$.

The exact solution to Eq. (38) is found numerically, using Eq. (41) for the fieutral atom flux. The resulting expression for the time-dependent electron density is substituted into Eq. (37). The resulting expression for the electron avalanche current density is then used to determine the diode shorting time.

The results of this calculation are presented in Figs. 17-23.

\section{STEADY-STATE SPACE-CHARGE-LIMITED}

\section{MAGNETICALLY INSULATED DIODE OPERATION}

Calculations for the anode current in the magnetically insulated diode have been made for two assumed distributions of reflexing ions. The first distribution, peaked at low reflex ion energies, is characteristic of a thin foil when the range is proportional to ion energy raised to the 1.8 power. Another proposed cathode about which the ions would reflex is an open mesh. We assume the resulting reflex ion energy distribution to be peaked at high energies. The assumed form of the normalized quadratic distribution is shown in Fig. 5. An open mesh cathode, with the ions either passing through without interaction, or being stopped and trapped by the mesh, would result in a delta-function reflex-ion energy distribution peaked at $\theta=1$. By including

Fig. 5. Energy spectra of the reflex ions at the cathode. The Eoil spectrum (solid curve) is peaked at low energy, and the mesh spectrum (broken curve) is peaked at bigh energy. The energy is expressed here as a (dimensionless) fraction of the diode potential $V_{0}$.

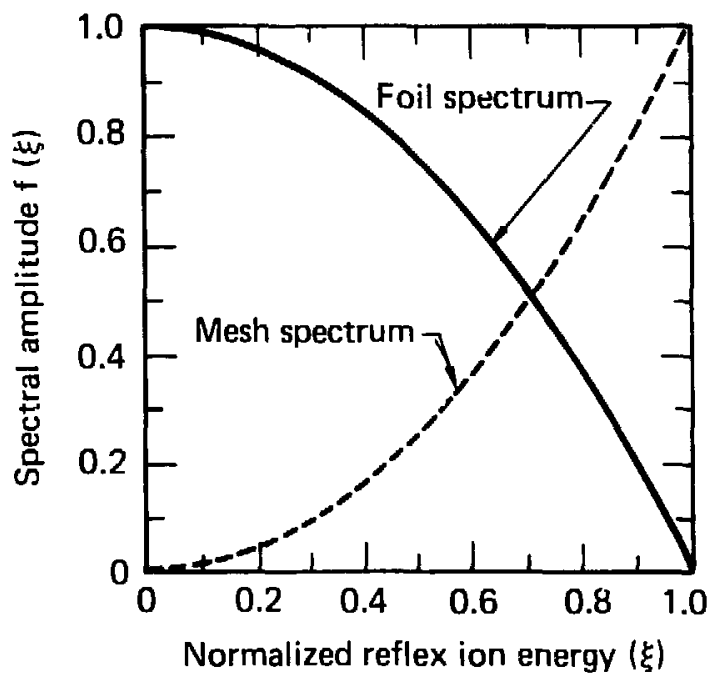


some scattering and energy loss by ions during transit, one may justify the quadratic distribution which is used.

The energy distribution of reflex ions at the cathode affects the equilibrium current density of positive ions at the anode. This is so because the reflex ions spread out between the cathode and anode. The greater the quantity of positive charge there is near the anode, the smaller the positive ion current density. In Fig. 6 we show the fraction of reflex ions whose energy exceeds a given value of $\xi=\phi / N_{0}$. This fraction is larger for the mesh distribution than for the foil distribution.

If the potential increased at a constant rate from the cathode to the anode (constant electric field), then Fig. 6 would also show the fraction of reflex ions extending a given distance from the cathode towards the anode. We see that at $\xi=0.8$, the mesh distribution has about 5 times the number of ions that the foil distribution has. This explains why the anode current (shcwn in Fig. 9) is smaller for the mesh than for the foil spectrum. This also explains why the mesh-current-to-foil-current ratio decreases for larger values of $\eta$, where $n$ is the average number of times an ion enters the cathode.

Figure 7 shows the potential distribution for a typical foil and mesh spectrum for the case of $\left(r B^{*} / \mathrm{r}_{\mathrm{mx}} \mathrm{B}\right)=0.1$. The potential distributions are similar to each other, although the electric field near the anode is smaller for the mesh spectrum. The potential varies smoothly from the anode to the cathode.

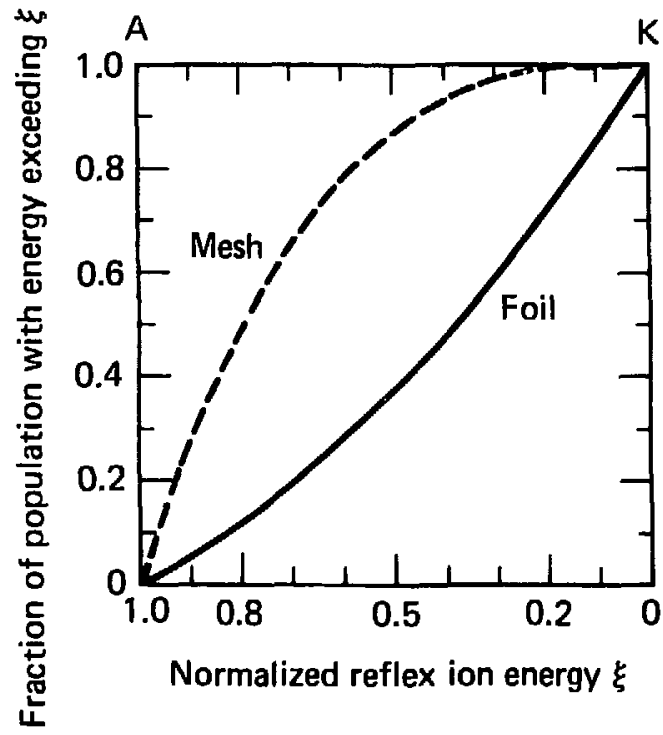

Fig. 6. Fraction of reflex ions whose energy exceeds a given amount. The energy is expressed here as a (dimensionless) fraction of the diode potential $\mathrm{V}_{0}$. The case of a foil cathode is represented by the solid curve, and that of a mesh cathode by the broken curve. 


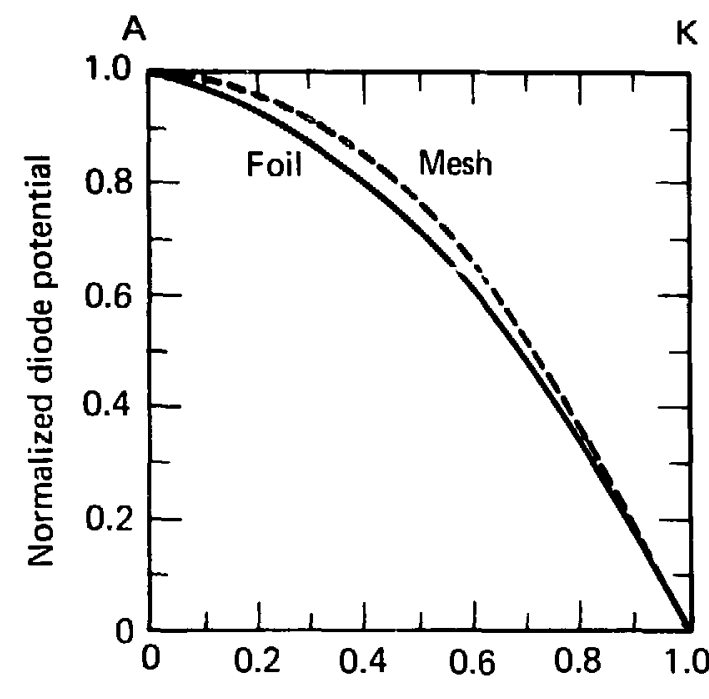

Fig. 7. Potential distribution within the diode for two typical cases. The solid curve is for a foil cathode, and the broken curve is for a mesh cathode. $n$, the number of times an ion enters the cathode, is equal to $20 . \mathrm{rB}^{*} / \mathrm{r}_{\mathrm{mx}} \mathrm{B}$ $=0.1$.

Fraction $\mathrm{z} / \mathrm{d}$ of electrode separation

Figure 8 shows the limits of the electric field distribution as the magnetic field is varied. $\eta$ is 20 for each case. The electric field in the anode region is plotted in units of $v_{0} / d$. The calculated electric fiela reproduces the Child-Langmui $r^{8}$ variation $\left(E \propto x^{1 / 3}\right)$ near the anode. Near the cathode (not shown in Fig. 8) the electric fields also approach zero. There is more variation of the electric fields near the cathode with variation of the magnetic field, as expected.

Fig. 8. Electric field distribution within the diode outside the cathode sheath of electrons. The limits are shown, between which the distribution ranges as the magnetic field is varied.

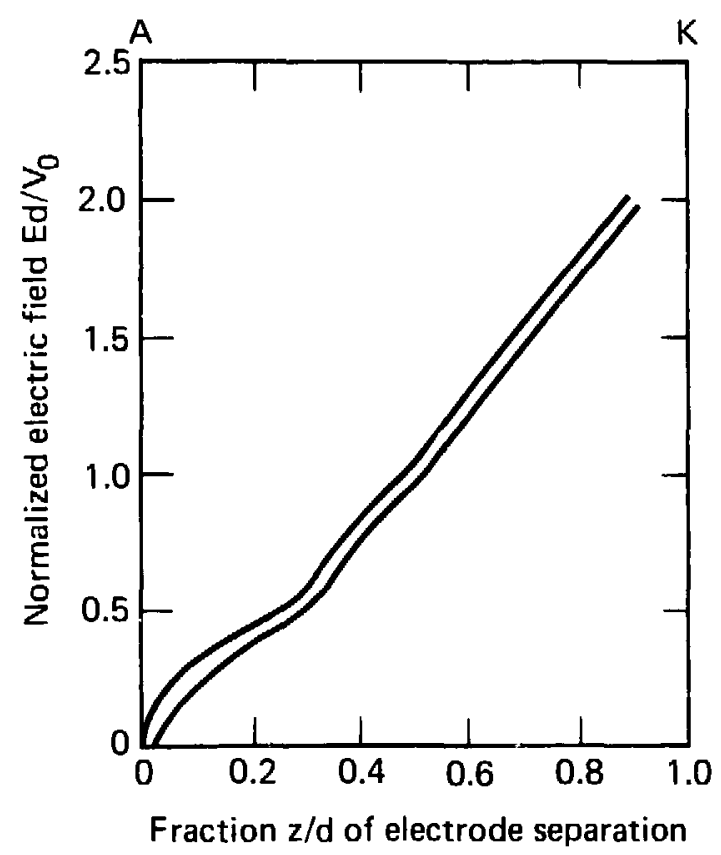

Fraction $\mathrm{z} / \mathrm{d}$ of electrode separation 
The anode and cathode current densities, divided by the respective ChildLangmuir vaiues, are shown in Figs. 9 and 10. The anode current density decreases with $\eta$, and is about independent of $\left(r B * / I_{m x} B\right)$. The anode current increases slightly for larger values of $r$, as the electron distribution then extends part way across the diode towards the anode.

In contrast, the anode current density varies strongly with reflex ion energy distribution and with $\pi$. The anode current density is about 3 times greater for the low-energy-peaked foil spectrum than for the high-energypeaked mesh spectrum. Indeed, the ion flux at the cathode is less for the mesh spectrum than for the case where reflexing is entirely absent. The reverse is true for the low-energy-peaked foil spectrum. For the foil spectrum, the ion flux with reflexing, at the cathode, exceeds the Child-Langmuir limit.

Figure 10 shows the electron current density at the cathode divided by the Child-Langmuir limit. The electron current density extends only a short distance into the diode gap before the magnetic field returns the electrons to the cathode. The electron current density at the cathode varies strongly with $\left(r B^{*} / r_{m x} B\right)$. The range of this variation is surprising, so an analytic study of Poisson's equation is reproduced in the appenaix. There the trend and numerical values of the computer solution are reproduced for the case of large

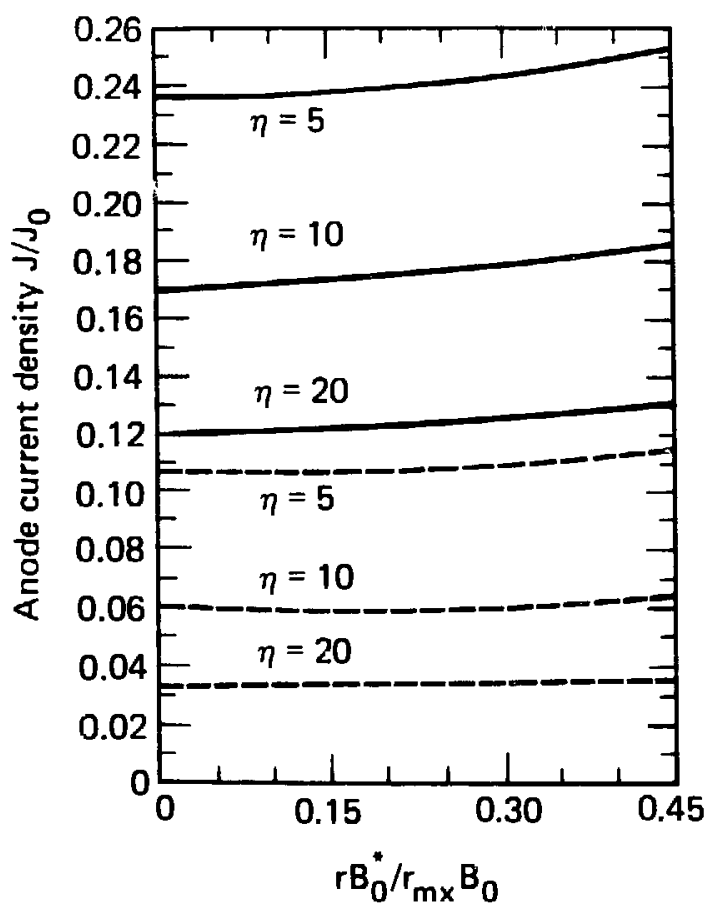

Fig. 9. Anode ion current density versus radius. $\eta$ is the number of times an ion enters the cathode. The anode current density is normalized to the Child-Langmuir current density. The radial coordinate is scaled to the magnetic field strength. The solid curves refer to a foil-cathode energy spectrum, and the broken curves, to a mesh spectrum. 


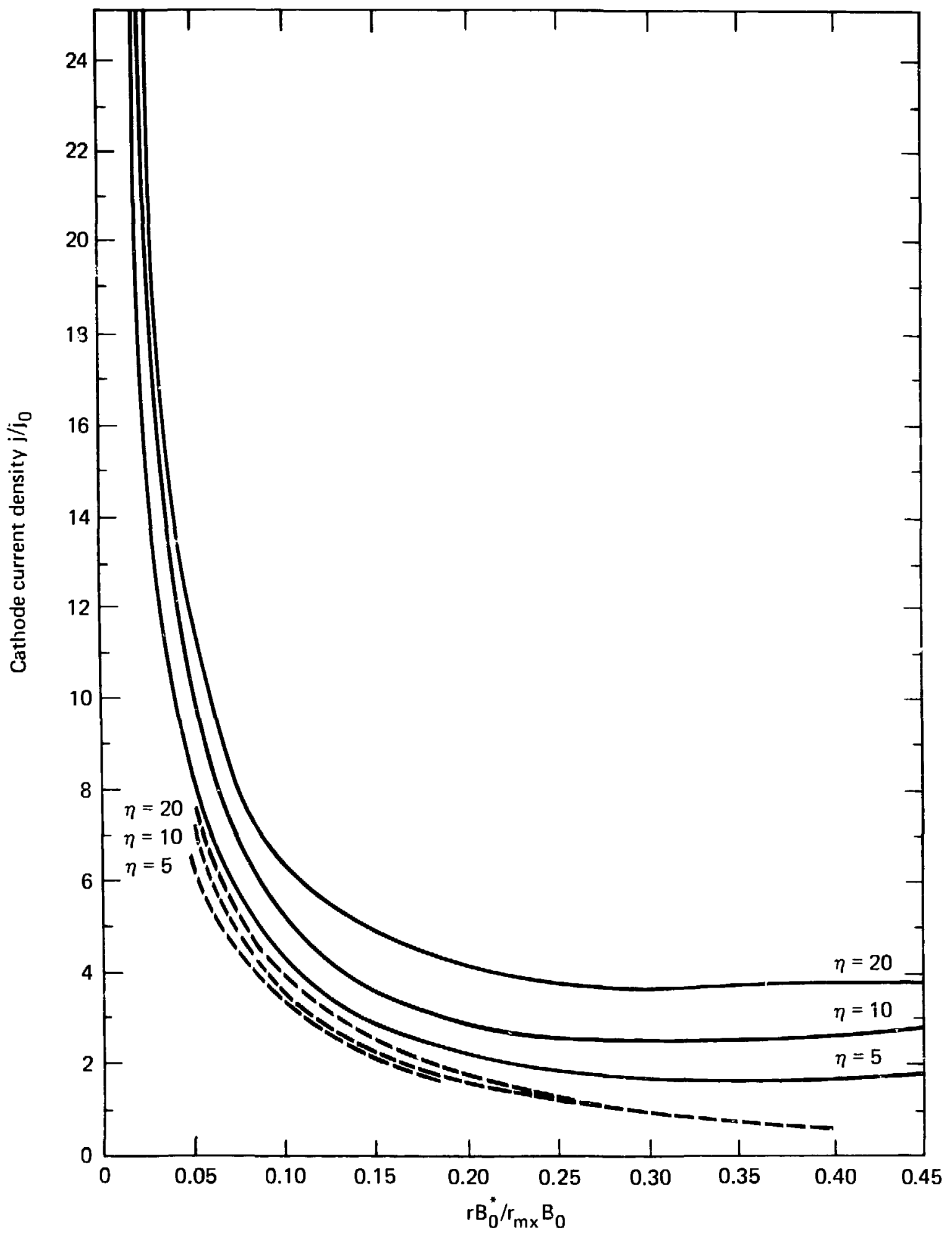

Fig. 10. Electron current density emitted by the cathode versus radius. jo is the Child-Iangmuir current density. The radial coordinate is scaled to the magnetic field strength. The solid curves refer to a foil cathode, and the broken curves refer to a mesh cathode. $\eta$ is the number of times an ion enters the cathode. 


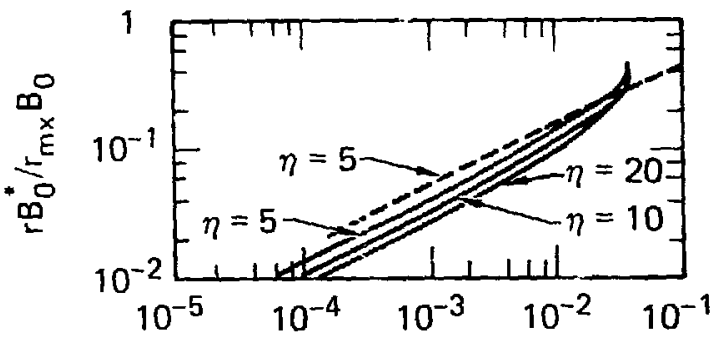

Boundary potential $u(b)$
Fig. 11. Dicde potential at the electron boundary, versus radius. Three values of $\eta$, the number of times an ion enters the cathode, are represented. The solid curves refer to a foil-cathode energy spectrum, and the broken curve, to a mesh spectrum. The radial coordinate is scaled to the magnetic field strength.

magnetic field. The electron current density also increases with the ion flux at the cathode, as expected.

For magnetic fields stronger than the insulating magnetic field, the electrons cannot cross the diode gap. How far an electron can get from the cathode depends on the potential distribution. The boundary potential u(b) where the electrons are turned by the magnetic field is plotted in Fig. II vorsus the magnetic field.

The variation of $u(b)$ is as expected: The greater the positive ion current density, the larger $u(b)$ is, and the further the electrons get from the cathode. The stronger the magnetic field, the smaller the boundary potential $u(b)$, and the closer the electron turning point is to the cathode.

This concludes the discussion of the steady-state space-charge flow far a magnetically insulated reflex ion diode. We turn now to the breakdown of the space-charge magnetically insulated flow.

\section{DIODE SHORTING TIME FROM CiIARGE-EXCHANGE AT ANODE}

In this section, we analyze the sequence of steps which cause the space-charge-limited flow to break down: Neutral atoms fill the diode gap, and become ionized by the reflexing ions and by electrons. The ionization of neutrals produces an electron avalanche whose current eventually equals and exceeds the anode current (which is due to positive ion flow). The rise of the avalanche electron current density to equal the ion current density determines the shorting time.

The density and flux of neutral atoms away from the anode are proportional to the space-charge-limited ion flux at the anode. See Fig. 9 for typical values of this ion flux. The neutral density $\rho$ at the cathode, from neutrals that have crossed the diode gap, can be found from Figs. 12-15. This 


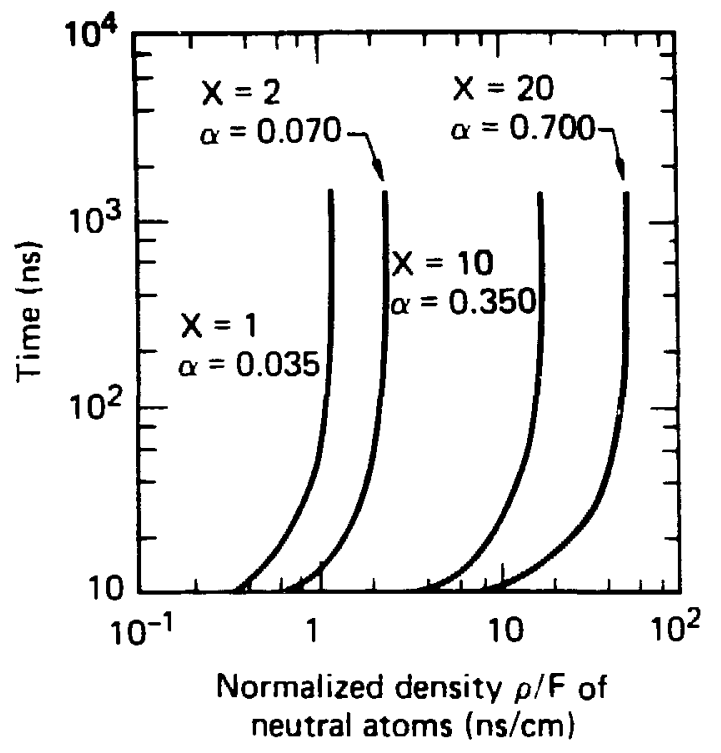

Fig. 12. Neutral atom (deuterium) density at cathode versus time. F is the anode ion flux. Curves are labeled by $X$ (the normalized position along the axial direction), and bv $\alpha$.

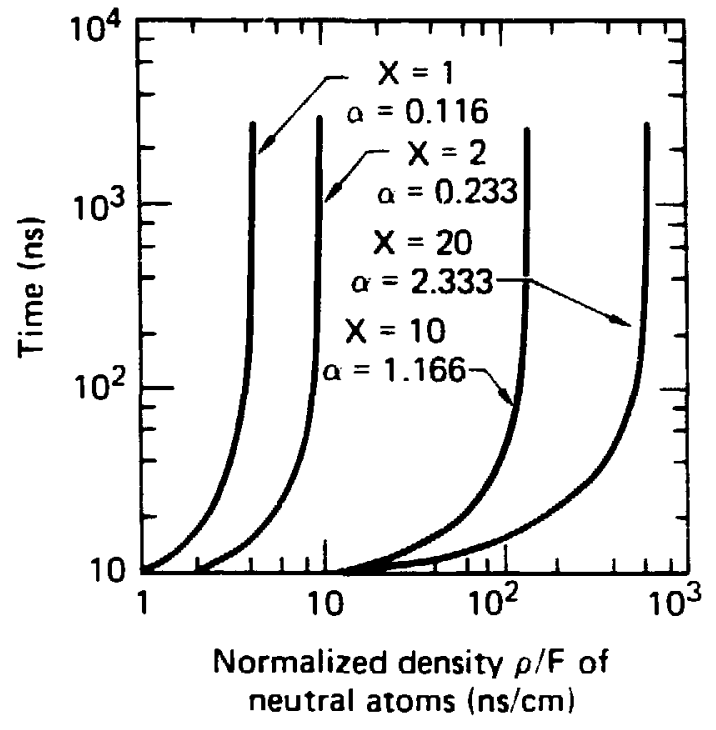

Fig. 13. Neutral atom density at cathode versus time. Curves are labeled by $X$ (the normalized position along the axial direction), and by $\alpha$.

density is found by integrating the distribution function, Eg. (26), over all velocities greater than $d / t_{0}$, where $t_{0}$ is the time required for the most energetic neutral to cross the diode $\mid \mathrm{Eg}$. (34)]. The ion flux $F$ has units of $1 /\left(\mathrm{cm}^{2}-\mathrm{ns}\right)$.

The neutral density is expressed in atoms $/ \mathrm{cm}^{3}$. Thus $\rho / F$ has units of $\mathrm{ns} / \mathrm{cm}$ and is plotted versus time (in nanoseconds) in Figs. 12-15. The timedependent neutral density at the cathode is plotted for various values of $x$ and $\alpha$. These were determined from the solution for the electric field, see

Fig. 14. Neutral atom density at cathode versus time. Curves are labeled by $X$ (the normalized position along the axial direction), and by $\alpha$.

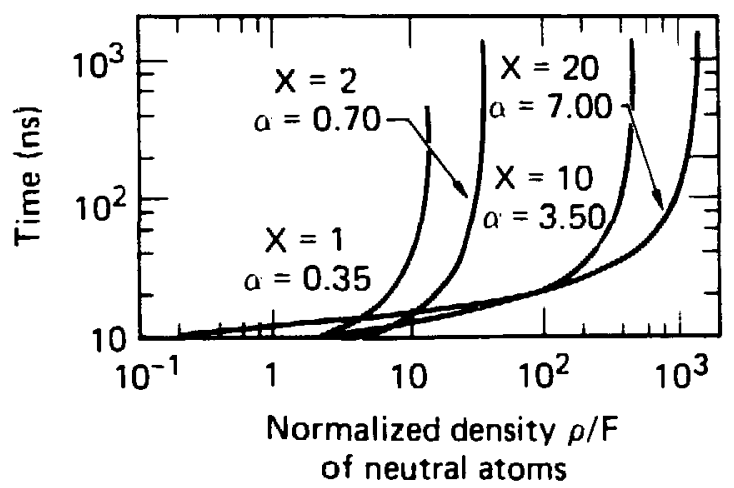




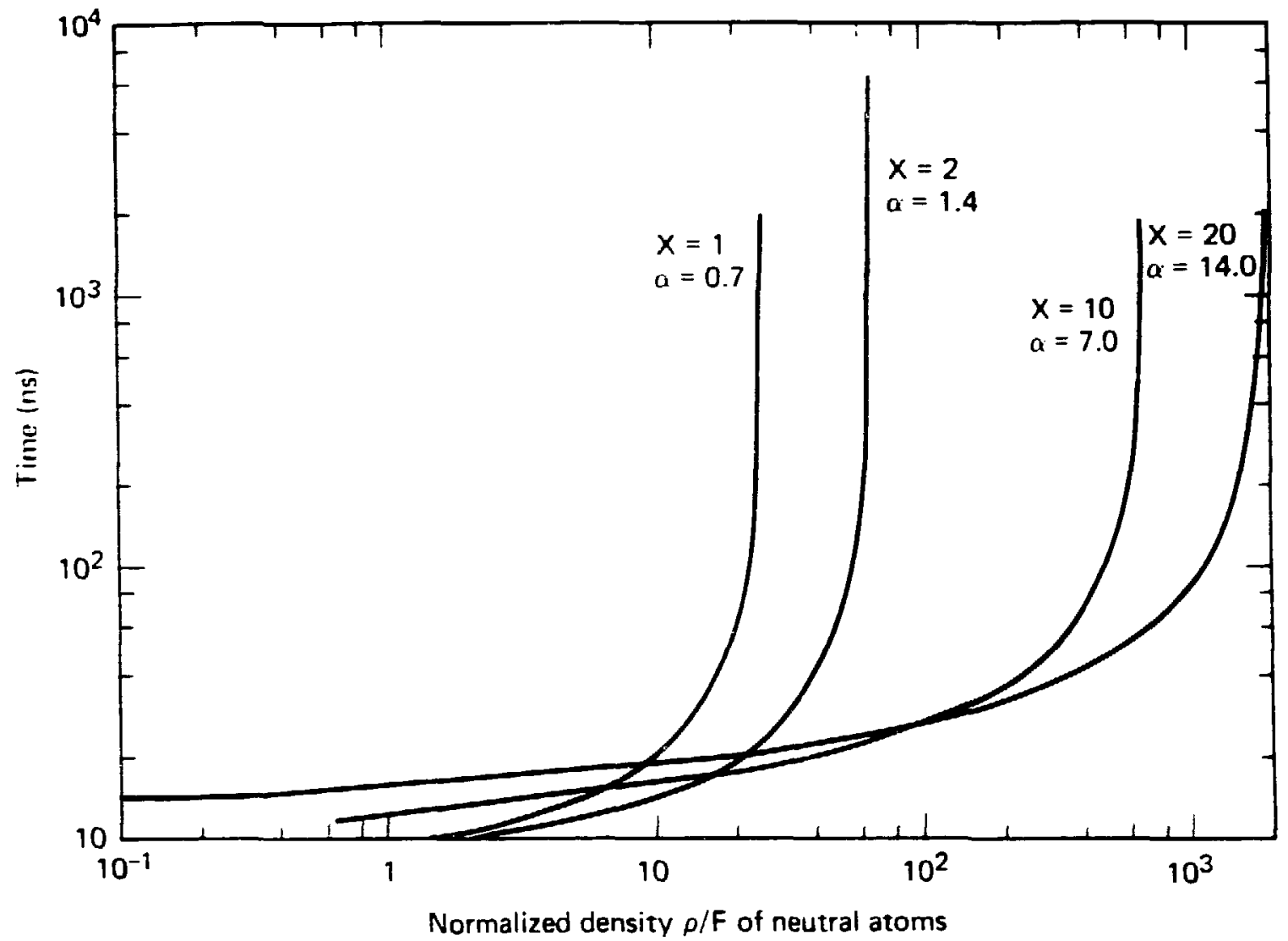

Fig. 15. Neutral atom density at cathode versus time. Curves are labeled by $x$ (the normalized position along the axial direction), and by $\alpha$.

Fig. 8, near the anode. The key factor is that the density at the cathode rises to within one half of its equilibrium value in about 50 ns for all cases studied. This $50 \mathrm{~ns}$ time for the neutrals to cross the diode provides a lower limit to the diode shorting time.

The anode flux $F$ can be expressed in terms of $\mathrm{J} / \mathrm{J}_{0}$, which may be read from Fig. 9. From Eq. (36) for the flux we have:

$$
F=\left(\frac{\mathrm{J}}{J_{0}}\right) 1.7 \times 10^{11}\left(\frac{\mathrm{ev}_{0}}{\mathrm{MeV}}\right)^{3 / 2} /(\mathrm{d} / \mathrm{cm})^{2} \frac{\text { ions }}{\mathrm{cm}^{2}-\mathrm{ns}} .
$$

The equilibrium densities are plotted versus $\alpha$ in Fig. 16. By "equilibrium," we mean the neutral atom density at the cathode after sufficient time of diode operation in the space-charge-limited mode. 
Fig. 16. Asymptotic steady-state neutral atom density at cathode versus $\alpha$. The curves are for constant $X$ values. $F$ is the anode ion flux. Note that $\alpha$ increases to the left.

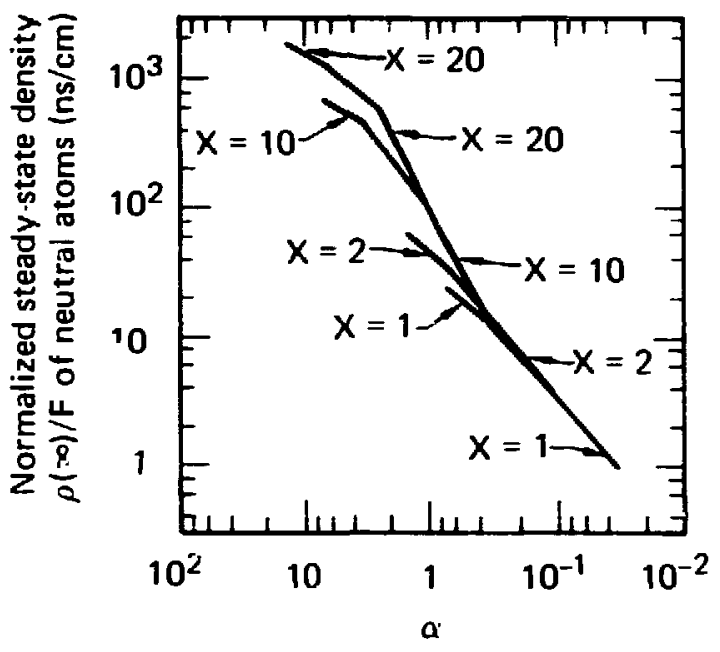

The equilibrium density can be obtained from integrating

$$
\rho(t)=\int_{d / t}^{x} d v g(x, v)
$$

where $\mathrm{g}$ is the distribution function given by Eq. (26). The asymptotic density becomes

$$
\rho / F=\sqrt{\frac{\pi \alpha M}{2 w_{0}}}\left(\sqrt{B} \text { erf } \sqrt{B X}-e^{-C B X} \text { erf } \sqrt{x}\right) / B C
$$

where "erf" stands for the error function.

The electron avalanche shorting time is plotted in Fig. 17 versus $\alpha$. The shorting time is approximately proportional to $\mathrm{e}^{-\alpha \pi / 2}$. The shorting time exceeds $10^{3}$ ns if $\alpha$ is about 4 or less.

In Fig. 18 we plot the shorting time as a function of magnetic field for various reflex ion spectra, with $\alpha$ equal to 4 .

The mesh spectrum is associated with a large shorting time because the reflex ion concentrations are smaller than for the cor-esponding foil spectrum. Ionization of neutrals by collision with ions is therefore less frequent, and electrons accumulate more slowly, prolonging the shorting time.

The curve shown in Fig. 17 suggests that for $\alpha=5$ or more, the shorting time is determined by the time the neutrals take to reach the cathode. This occurs at about 50 to 100 ns into the space-charge-limited mode, as seen in Figs. 14 and 15 . 


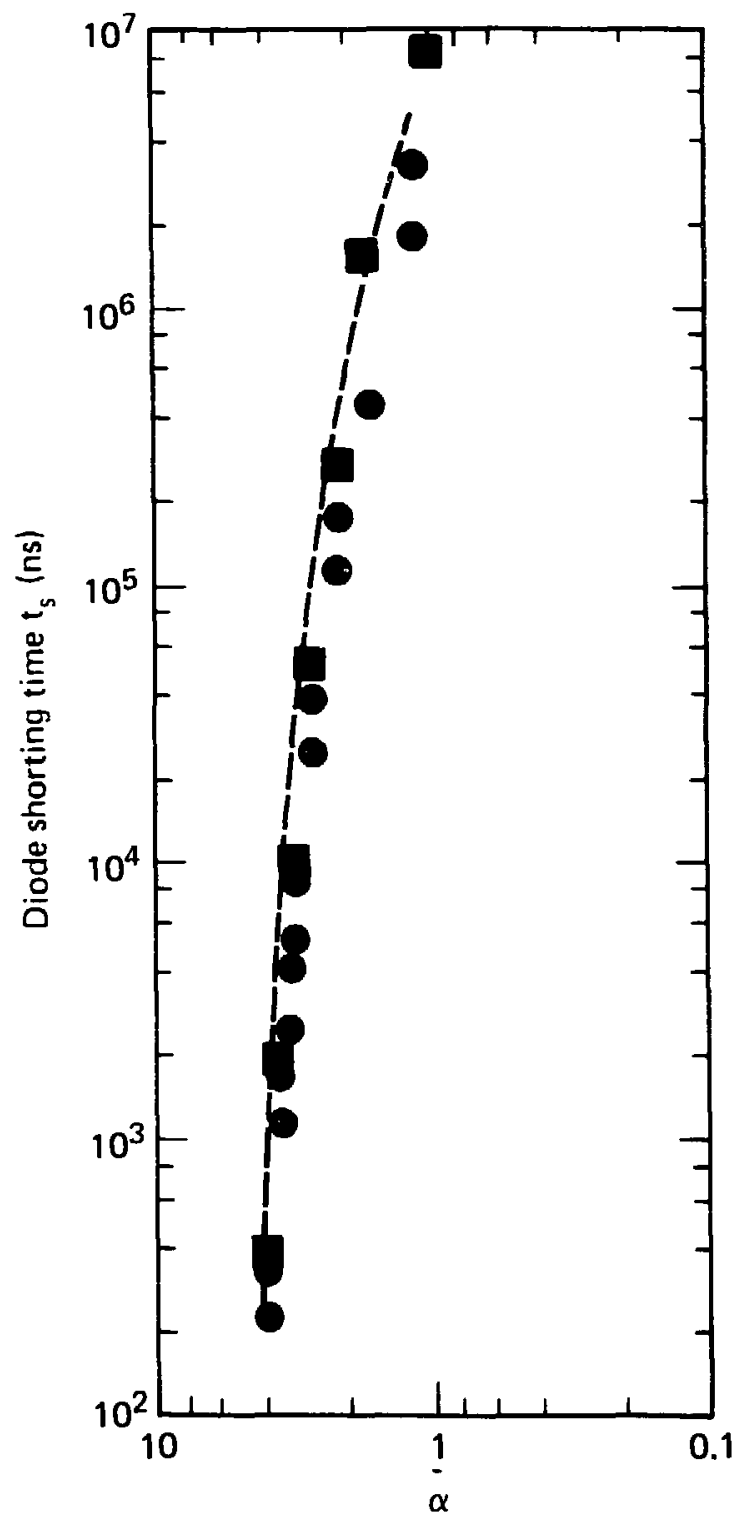

Fig. 17. Diode shorting time versus $\alpha$. The solid squares represent the case where $\eta=20, r B^{*} / r_{m x^{B}}$ $=0.4$, and the cathode is foil. For the solid circles, $\eta=5$, $\mathrm{rB} * / \mathrm{I}_{\mathrm{mx}} \mathrm{B}=0.1$, and the cathode is foil. For the broken line, $n=5$, $r B^{*} / r_{m x} B=0.1$, and the cathode is mesh.

The scaling of the shorting time is shown in Figs. 19-23. Figure 19 shows a semilog plot of the shorting time versus $\alpha$. This shows that shorting time is proportional to $e^{-\alpha \pi / 2}$. In Figs. 20 and 21 , we show the shorting time versus the diode potential $v_{0}$, holding the diode gap $d$ constant and the magnetic field constant. The curves for various reflex ion spectra, and for selected values of $\alpha$, are all parallel; these curves show that shorting time is inversely proportional to $v_{0}$.

In Figs. 22 and 23, the shorting time is plotted versus the diode separation, holding the potential difference across the gap fixed at 2.044 


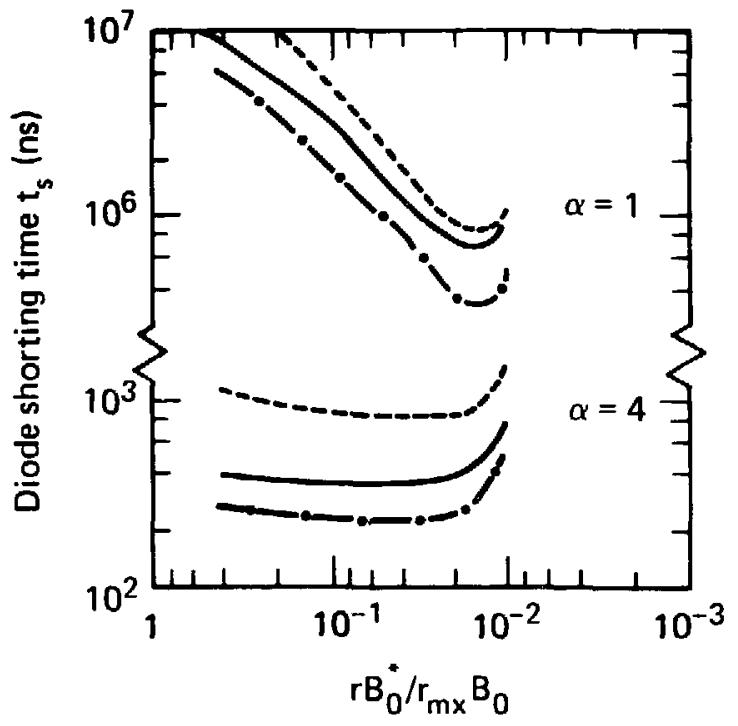

Fig. 18. Diode shorting time versu: radius, at constant $\alpha$. For the upper curves (dashed), $\eta=5$, and the cathode is mesh. For the middle curves (solid), $\eta=20$, and the cathode is foil. For the lower curves (dot-dash-dot), $\Pi=5$, and the cathode is foil.

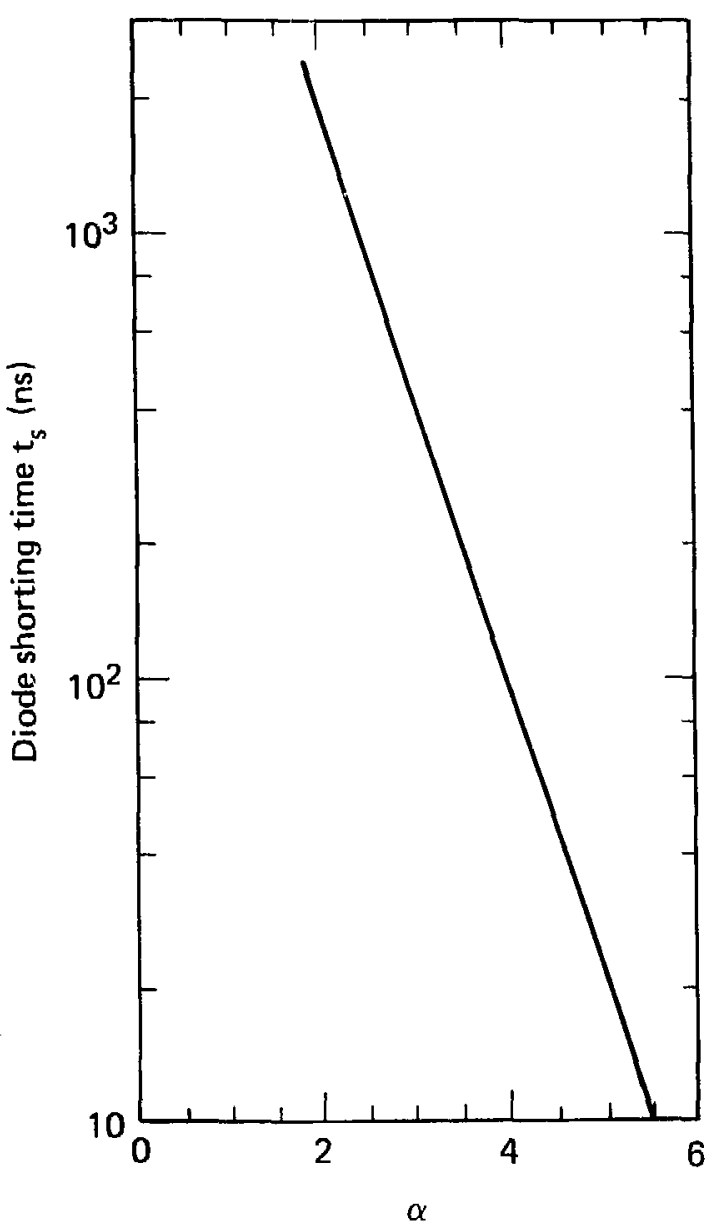

Fig. 19. Scaling of shorting time versus $\alpha$. Diode gap is fixed at $0.7 \mathrm{~cm}$, for a mesh spectrum. The diode potential $\mathrm{V}_{0}$ is fixed at $4 \mathrm{mc}^{2} / \mathrm{e}$, and $\mathrm{rB}^{*} / \mathrm{r}_{\mathrm{mx}} \mathrm{B}=0.1$. 


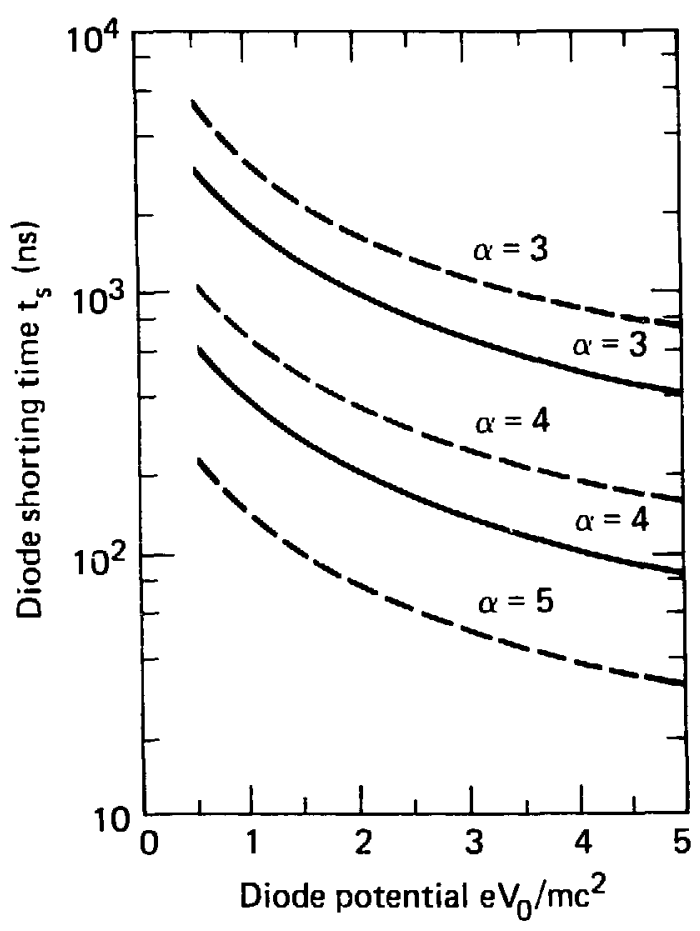

Fig. 21. Shorting time versus gap potential. Diode gap is fixed at $1.0 \mathrm{~cm}$. , and magnetic field is fixed. Broken lines correspond to a mesh-cathode energy spectrum, and solid lines, to a foil spectrum.
Fig. 20. Shorting time versus gap potential. $n$ is 20 , diode gap is fixed at $1.0 \mathrm{~cm}$., and $\mathrm{rB}^{*} / \mathrm{r}_{\mathrm{mx}} \mathrm{B}^{\mathrm{B}}=$ 0.1 . Broken lines correspond to a mesh-cathode energy spectrum, and solid lines, to a foil spectrum.

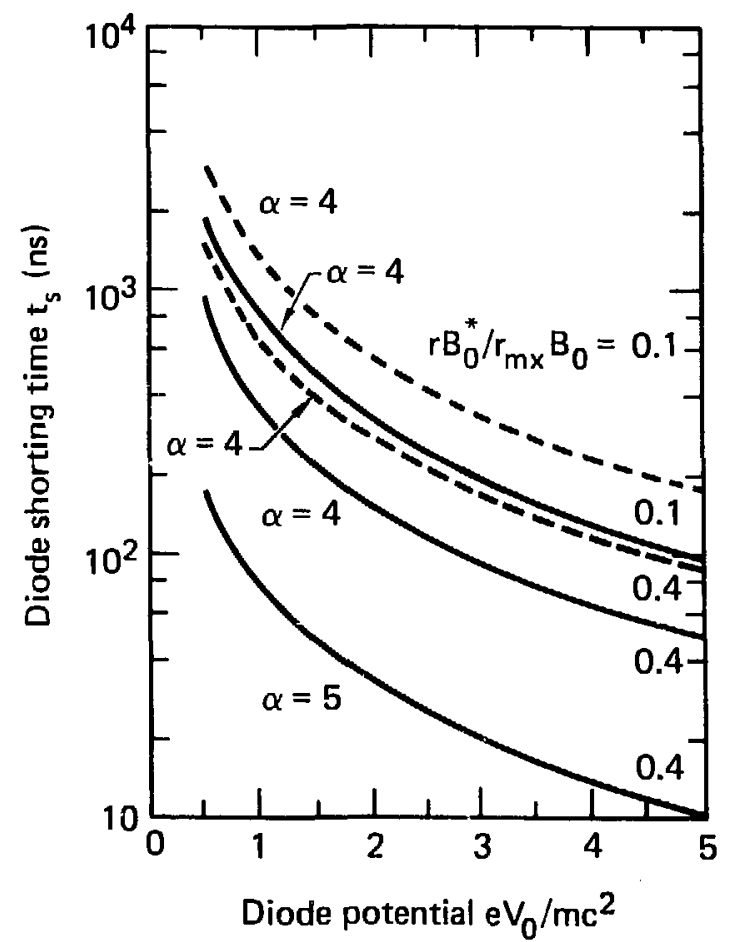




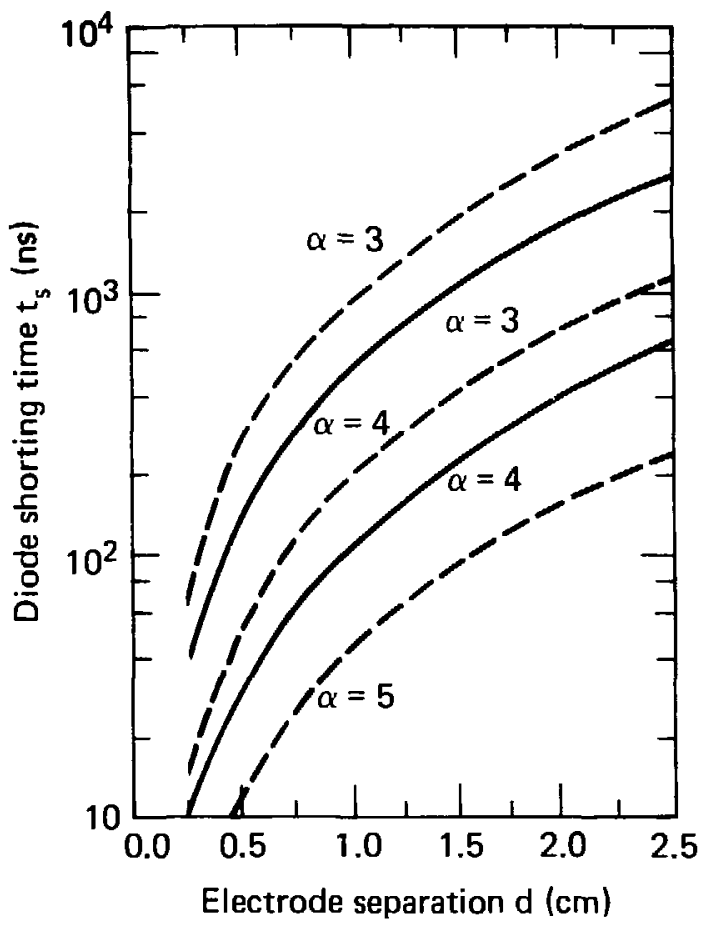

Fig. 22. Shorting time versus gap separation. $\mathrm{v}_{0}=4 \mathrm{mc}^{2} / \mathrm{e}$, $\mathrm{rB}^{*} / \mathrm{r}_{\mathrm{mx}} \mathrm{B}=0.1$. Broken lines correspond to a mesh-cathode energy spectrum, and solid lines, to a foil spectrum.

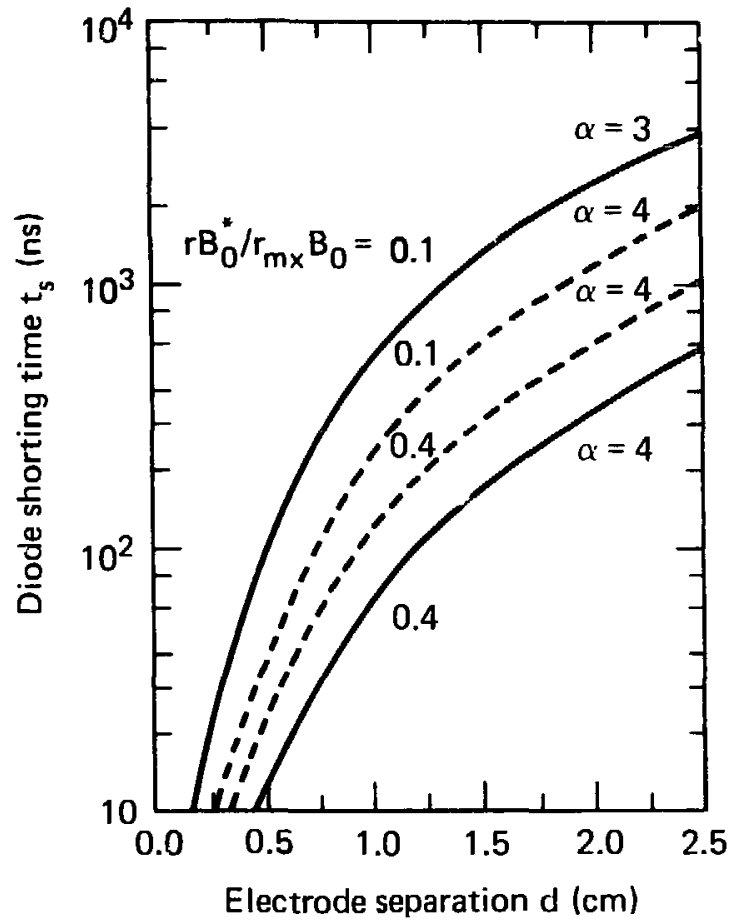

Fig. 23. Shorting time versus gap separation. Magnotic field is held constant, and $\mathrm{v}_{0}=4 \mathrm{mc}^{2} / \mathrm{e}$.

MeV. The curves for various $\alpha$ are all parallel and show that the shorting time is proportional to $\mathrm{d}^{2}$.

These curves have all used the deuteron mass for the ion leaving the anode. The times will be reduced by a fistor of 2 if protons leave the anode.

\section{POSSIBLE FUTURE MODIFICATIONS}

In this work we have calculated the electron avalanche shorting time from a charge exchange mechanism at the anode. Static neutral atoms undergo charge exchange with accelerated ions resulting in drifting neutrals that undergo no further interaction.

Future work should include charge exchange of the drifting neutral atoms with the reflexing ion distributions. Such subsequent re-exchange would slow or reverse the direction of the neutrals. In either event the flow of neutrals to the cathode would be slowed, increasing the shorting time. 
Secondarily, the time-dependence of the diode potential should be included in a quasistatic approximation. Here the full potential was assumed turned on at $t=0$. The 20-ns or so rise time of the diode potential should be included in future work. As a result of including such a rise time for the potential, a [see Eg. (27)] would vary with time because the anode electric field would wary with time. Such a time variation of a might significantly change the shorting time. The variation of the electric field with position should be included. The distribution function used assumed an average electric field. Finally, an approximation which takes into account the effect of the electric field on the lonization and charge exchange cross sections ${ }^{9}$ can be included.

Large electric fields tend to reduce the charge exchange cross section and to enhance the ionization cross section. The first change would increase the shorting time while the enhanced ionization cross section might decrease the shorting time. Whether the electric field would cause significant changes, or in which direction, is not clear.

Rnowledge of the neutral atom distribution near the anode surface would be very helpful. 
1. D. S. Prono, J. M. Creedon, I. Smith, and N. Bergstrom, "Multiple Reflections of Electrons and the Possibility of Intense Positive-Ion Flow in High $V / Y$ Diodes," J. Appl. Phys. 46, 3310-3319 (1975).

2. T. J. Orzechowski and G. Bekefi, "Current Flow in a High-Voltage Diode Subjected to a Crossed Magnetic Field," Phys. Fluids 19, 43-51 (1976).

3. J. M. Creedon, J. D. Smith and D. S. Prono, "Method of Generating Very Intense Positive-Ion Beams," Phys. Rev. Lett. 35, 91-94 (1975).

4. M. A. Greenspan, R. Pal, D. A. Hammer, and S. Humphries, Jr., "An Applied-B Magnetically Insulated Ion Diode," Appl. Phys. Lett. 37, 248-250 (1980).

5. D. S. Prono, H. Ishizuka, E. P. Lee, B. W. Stallard, and W. C. Turner, Charge-Exchange Neutral-Atom Filling of Ion Diodes: Its Effect on Diode Performance and $A-K$ Shorting, Lawrence Livermore National Laboratory, Livermore, CA, UCRL-84438 (1980). This has been submitted to Journal of Applied Physics (1981).

6. J. W. Shearer, Space Charge Equation Calculations for the Reflex Triode, Lawrence Livermore National Laboratory, Livermore, CA, UCRL-52129 (1976).

7. R. L. Freeman and E. M. Jones, "Atomic Collision Processes in Plasma Physics Experiments," URAEA Research Group, Culham Laboratory, Abingdon, Berkshire, England, CLM-R 137 (1974). Available from H. M. Stationery office. I gratefully acknowledge W. C. Turner for calling this report to my attention.

8. I. Langmuir, "The Interaction of Electron and Positive Ion Space Charges in Cathode Sheaths," Phys. Rev. 33, 954-989 (1929).

9. R. E. Olson and A. D. MacKellar, "Ion Collisions with Rydberg Atoms in Strong Electric Fields," Phys. Rev. Lett. 46, 1451-1454 (1981). 
Let us consider the magnetic field to be so strong that the electron current is nonrelativistic, and the potential where the electrons are is small compared to $\mathrm{mc}^{2}$. Then Eq. (2I) becomes:

$$
\begin{aligned}
& \left(\frac{d u}{d x}\right)^{2}=\frac{-4 J}{J_{0}}[1-\sqrt{1-u}+s(0)-s(u)]+\frac{4 j}{j_{0}} 2 \sqrt{u} \\
& \equiv \frac{-4 J}{J_{0}} k u+\frac{4 j}{j_{0}} 2 \sqrt{u} .
\end{aligned}
$$

For u small compared to 1 , taking the square root, we obtain:

$$
\frac{d u}{d x} \approx \sqrt{\frac{8 j}{j_{0}}} u^{3 / 4}
$$

Integrating $\mathrm{Eq} .(\mathrm{A}-3)$ we obtain

$$
\begin{aligned}
& 1-x=\sqrt{\frac{j_{0}}{8 j}} \frac{4}{3} u^{3 / 4} . \\
& \text { Using } A^{\prime} \equiv \mathrm{rB}^{\star} / r_{\mathrm{mx}}{ }^{B},
\end{aligned}
$$

with Eq. (17), and neglecting $U^{2}$ compared to $U$, we have

$$
1-x=A^{\prime} U^{1 / 2} /\left(1+e v_{0} / 2 m c^{2}\right)^{1 / 2}
$$

Equation (19), in the $U \rightarrow 0$ limit becomes

$$
\left(\frac{J}{J_{0}}\right)^{1 / 2}=\int_{0}^{1} \frac{d u}{4[\sqrt{1-U}+S(U)]}=T I(0) .
$$

By equating electric fields inside and outside the electron boundary, using Eq. (19) and Eq. (A-3), the electron current density becomes:

$$
\left(\frac{j}{j_{0}}\right)=\frac{J}{J_{0}} \frac{[1+S(0)]}{2 \sqrt{U}} .
$$


Using Egs. (A-5, A-6, A-7) in Eq. (A-4), we find

$$
\sqrt{\delta}=\frac{A^{\prime} T I(0) \times 1.5}{\left(1+e V_{0} / 2\right)^{1 / 2}}
$$

This reflects the large magnetic field (small $A^{\prime}$ ) behavior of $U$ shown in Fig. 11. And using Eq. $(A-8)$ in Eq. (A-7), we see the cathode current density varies inversely with $A^{\prime}$, as seen in the computer solution shown in Fig. 10 . 